\title{
Secure Communications in High Speed Fiber Optical Networks Using Code Division Multiple Access (CDMA) Transmission
}

\section{1-ERD-002}

R.J. Welty, C.E. Reinhardt, Y. Du*+, N.P. Kobayashi, V.R. Sperry, I.Y. Han, E.M. Behymer, S.W. Bond, and S.J. Yoo+

\section{* LLNL SEGRF Fellowship Student + University of California, Davis}

In collaboration with Prof. Ben Yoo's

Optical Switching \& Communications Systems Laboratory at the University of California, Davis

\begin{tabular}{|c|c|}
\hline \multicolumn{2}{|c|}{ Project Funding } \\
\hline$\overline{\text { FY01 }}$ & $\$ 488 \mathrm{k}$ \\
\hline FY02 & $\$ 397 k$ \\
\hline FY03 & $\$ 200 \mathrm{k}$ \\
\hline Tota & $\$ 1,085 k$ \\
\hline
\end{tabular}

February 17, 2004 
This document was prepared as an account of work sponsored by an agency of the United States Government. Neither the United States Government nor the University of California nor any of their employees, makes any warranty, express or implied, or assumes any legal liability or responsibility for the accuracy, completeness, or usefulness of any information, apparatus, product, or process disclosed, or represents that its use would not infringe privately owned rights. Reference herein to any specific commercial product, process, or service by trade name, trademark, manufacturer, or otherwise, does not necessarily constitute or imply its endorsement, recommendation, or favoring by the United States Government or the University of California. The views and opinions of authors expressed herein do not necessarily state or reflect those of the United States Government or the University of California, and shall not be used for advertising or product endorsement purposes.

This work was performed under the auspices of the U.S. Department of Energy by University of California, Lawrence Livermore National Laboratory under Contract W-7405-Eng-48. 


\section{Table of Contents}

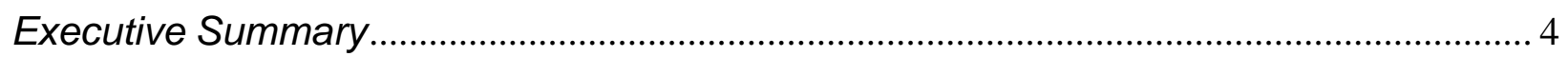

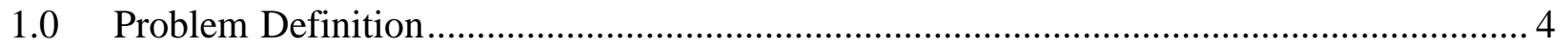

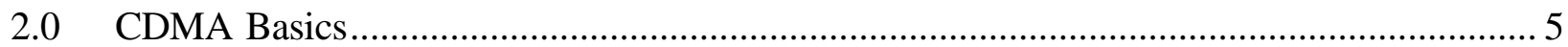

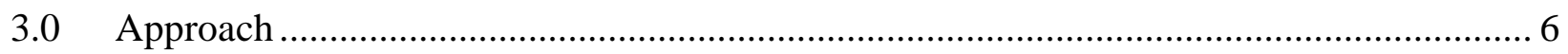

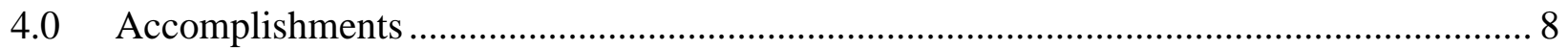

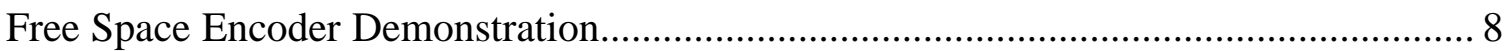

FY02 - Transisition to Integrated device Research...................................................... 12

Free Space (Table Top) Encoder Demonstration............................................................. 12

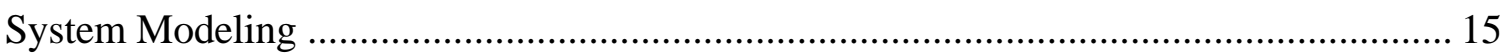

Monolithically Integrated Technology...................................................................... 16

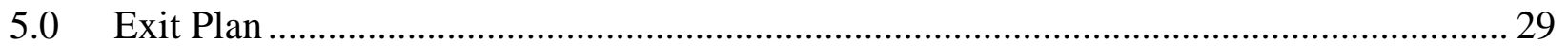

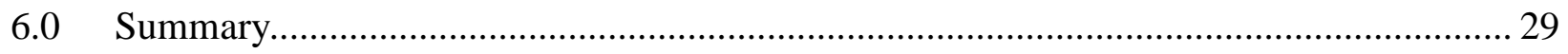




\section{Executive Summary}

This project is focused on the development of advanced components and system technologies for secure data transmission on high-speed fiber optic data systems. This work capitalizes on 1) a strong relationship with outstand ing faculty at the University of CaliforniaDavis who are experts in high speed fiber-optic networks, 2) the realization that code division multiple access (CDMA) is emerging as a bandwidth enhancing technique for fiber optic networks, 3) the realization that CDMA of sufficient complexity forms the basis for almost unbreakable one-time key transmissions, 4) our concepts for superior components for implementing CDMA, 5) our expertise in semiconductor device processing and 6) our Center for Nano and Microtechnology, which is where the majority of the experimental work was done.

Here we present a novel device concept, which will push the limits of current technology, and will simultaneously solve system implementation issues by investigating new state-of-the-art fiber technologies. This will enable the development of secure communication systems for the transmission and reception of messages on deployed commercial fiber optic networks, through the CDMA phase encoding of broad bandwidth pulses. CDMA technology has been developed as a multiplexing technology, much like wavelength division multiplexing (WDM) or time division multiplexing (TDM), to increase the potential number of users on a given communication link. A novel application of the techniques created for CDMA is to generate secure communication through physical layer encoding. Physical layer encoding devices are developed which utilize semiconductor waveguides with fast carrier response times to phase encode spectral components of a secure signal. Current commercial technology, most commonly a spatial light modulator, allows phase codes to be changed at rates of only 10's of Hertz $(\sim 25 \mathrm{~ms}$ response). The use of fast (picosecond to nanosecond) carrier dynamics of semiconductors, as opposed to field dynamics of liquid crystal molecules, enable phase codes at $\mathrm{GHz}$ rates. The semiconductor arrayed waveguide grating (AWG) is the building block of the encoder/decoder device. A monolithically integrated AWG is developed in this LDRD. Using this building block, the AWG can be integrated with phase modulators to create temporally varying phase codes; this allows superior physical level encoding technology.

The breadth of this project is wide, covering a free space optic demonstration (large optic at the meter scale) of the encoding system. This was done as a proof-of-principal exercise and to investigate the time varying phase codes ("locks" and "keys"). Then a monolithically integrated AWG implemented at the millimeter was investigated. The monolithically integrated AWG has the same functionality as the table top free space optic but reduced down in size to be easily embedded in fiber optic networks.

\subsection{Problem Definition}

High-speed, high-capacity fiber-optic communications networks, which allow multiple users to access the same network simultaneously by sharing the same transmission medium, have proliferated for long distance, metropolitan and local area communications systems. Recently a rapid expansion into wavelength division multiplexing (WDM) systems, which use multiple wavelengths to increase capacity, is taking place. In addition, it has been recognized that code division multiple access (CDMA) techniques, which were originally developed for wireless communications, can also be utilized to further multiplex users on an optical fiber [1][2]. These 
fiber networks will be the most important medium for the transfer of information worldwide for the foreseeable future. In many applications, it is very important to be able to transmit information over such fiber systems securely. We at LLNL have developed a concept that allows transmission of secure signals with optical CDMA (O-CDMA) formats which utilize complex, time-varying codes.

Our concept is to utilize the techniques of O-CDMA, where each user imprints their own message bits with their own unique code, to spectrally phase modulate (lock) a broadband transmitted optical pulse, which can only be decoded by the complementary phase modulation (key) at the receiver end. Our realization, the basis for this work, is that by varying a complex modulation code dynamically, on each transmitted pulse if necessary, the data stream can be made virtually immune to interception by unauthorized third parties not in possession of the correct time varying key.

Attempts to utilize CDMA in the past for secure communications have failed due to inferior component technology unsuited for the formation and tracking of sufficiently complex, temporally varying codes. In fact, one such previous research effort, supported by various US Government agencies, utilized parallel fiber (Sagnac) loops with slow mechanical fiber stretchers to introduce phase delays on a transmitted broadband pulse. The component technology and system implementation which we propose here will allow much faster phase code changes, by using novel integrated solid state waveguides and modulators, and the subsequent tracking and decoding of these signals after propagation across long distance fiber networks.

\subsection{CDMA Basics}

Code Division Multiple Access or CDMA in its traditional implementation is a form of "spread spectrum," a family of digital communication techniques that have been used in military applications for many years. The core principle of spread spectrum is the use of noise-like carrier waves, and, as the name implies, bandwidths much wider than that required for simple point-topoint communication at the same data rate.

CDMA is well known in the commercial wireless arena, where carriers are faced with the challenge of increasing the number of potential users within the FCC allocated frequency bands. Traditional methods of increasing the number of users within these frequency ranges utilized either user assigned frequency channels or time slots or both. These techniques suffer from the limitation on the number of simultaneous users. CDMA, and specifically "spread spectrum," offered a better solution to this capacity problem. The low bandwidth voice signals from cellular phone users can be temporally modulated by high-speed pseudo-random codes and the low frequency content of the signal is "spread" to cover the allocated portion of the EM spectrum. Each CDMA code is specific to a given user and all additional users within range only add to the average noise background. The received encoded signal is separated from the background noise by taking the autocorrelation of the complex conjugate of the original modulation code and the received signal. Figure 1 gives a simple graphical explanation of spread spectrum CDMA encoding technology.

Simply stated, "spread spectrum" uses a temporal modulation of the signal to "spread" the signal in the frequency domain ("spectrum"). Now consider the frequency domain analog, which we will call "spread time". "Spread time" uses a spectral modulation of a broad bandwidth pulse to spread the pulse in time. Spectral modulation can be achieved by varying the phase of each wavelength element of a broadband transmission signal. Figure 2 gives a simple graphical implementation. 


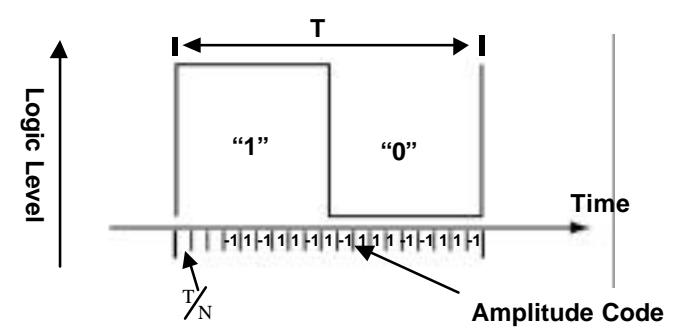

Figure 1: "Spread Spectrum" uses high-speed temporal modulation to spread the frequency content of a low bandwidth signal.
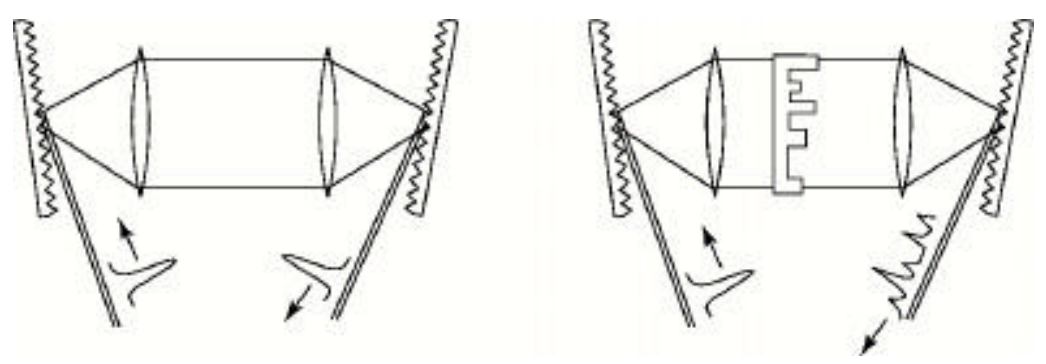

Figure 2: A short pulse of light containing a broad spectral content can be dispersed using a diffraction grating and a lens. At the Fourier plane, a phase mask can be inserted creating different phase delays for each spectral slice of the pulse thereby encoding the pulse.

The advantage of spectral phase encoding as opposed to temporal amplitude coding is that the "time spread" signals maintain their high-speed nature throughout the system; i.e. no signal bandwidth is sacrificed for the coding, a problem which worsens with increasing code complexity. Consider again Figure 1 and notice that the high-speed amplitude code for conventional spread spectrum indicated at the bottom of the figure has a speed necessarily many times that of the information signal. If, for example, the information signal has an inherent speed of $10 \mathrm{Gbps}$ (gigabits per second), and the code length of the amplitude modulation scheme is 100 , then the digital electronics used to encode the information bits must operate at clock speeds of $100 * 10 \mathrm{Gbps}=1 \mathrm{Tbps}$ (terabits per second). The proposed spectral phase encoding methodology does not suffer from the loss of temporal resolution. If the signal starts at $10 \mathrm{Gbps}$, the fastest electronics required to spectrally phase encode the signal uses the same clock speed of 10 Gbps.

\subsection{Approach}

The work done in this LDRD has developed new approaches and technologies to allow secure communication through the phase encoding of data transmitted across optical fiber networks. CDMA technology has been developed as a multiplexing technology, much like wavelength division multiplexing (WDM) or time division multiplexing (TDM), to increase the potential number of users on a given communication link. A novel application of the techniques created for CDMA is to generate secure communications through physical layer encoding. Our approach is based upon the work of Professor Heritage (UC Davis, collaborator on this project) 
and Professor Weiner (1988) in which a broadband, ultra fast signal from a mode locked (Erdoped fiber) laser is first dispersed in wavelength and then encoded in a dispersion matched grating-lens pair prior to insertion into a transmission fiber [3](see Figure 2). A phase mask, inserted in the Fourier plane of the grating lens pair, places a pre-programmed phase delay (the key) on each pixel or wavelength element of the ultra short pulse. The result is a pulse, stretched in time and reduced in amplitude, by up to two orders of magnitude, which can co-propagate with standard network traffic. The optical pulse is recovered at the receiver end by a complementary phase mask that removes the phase imprint at the input end and thereby restores the amplitude of the input pulse (minus passive system losses).

Current commercial technologies allow phase codes to change at slow rates $(40 \mathrm{~Hz})$ limited by the response time of liquid crystal modulators $(25 \mathrm{~ms})$. We have been developing superior physical level integrated encoding devices using Indium Phosphide (InP) arrayed waveguide gratings (AWG) and modulators. By employing fast (picosecond to nanosecond) carrier dynamics of semiconductors, as opposed to field dynamics of liquid crystal molecules, temporally varying, spectrally sliced, phase codes can be generated and reconfigured at $\mathrm{GHz}$ rates. Furthermore, through the careful design of phase modulation codes, the power spectrum of a transmitted pulse can be spread sufficiently such that third parties will experience the signal as a low level noise background or "interference" signal.

This LDRD has been focused on designing and building an innovative device expected to enable rapidly $(\mathrm{GHz})$ reconfigurable encoders and decoders. The device we have been developing is a variation of an arrayed-waveguide-grating (AWG) but with the added functionality of phase modulators. The AWG device is currently being implemented for WDM fiber optic networks for wavelength routing and is commonly referred to as a Dragone [4] coupler. In the telecom case, many inputs are coupled to single mode waveguides of various lengths through a broad diverging planar waveguide. The outputs of these different length waveguides have constructive interference at the output waveguides. The routing functionality is dependent upon the wavelength and input position of the AWG and predetermines the output waveguide, characteristics useful for WDM add/drop multiplexing and routing. For our device, we replace the many input waveguides with a single input guide, carrying a broadband signal; the output waveguides then collect a spectral slice of the input pulse bandwidth.

The monolithic reconfigurable spectral phase modulator shown below in Figure 3 essentially has the same functionality as the apparatus of Figure 2. The left most AWG in Figure 3 has the same functionality as the leftmost diffraction grating and lens of Figure 2 and creates a Fourier plane at the center of the device where the phase plate has been replaced by waveguide electro-optic phase modulators. The right AWG on the device shown in Figure 3 reassembles the spectrally phase encoded pulse just like the right diffraction grating and lens of Figure 2.

Rapid reconfiguration of the phase codes can be achieved in the Indium Phosphide (InP) material system, at excitation wavelengths of $\lambda=1.55 \mu \mathrm{m}$, by exploiting electro-optical effects in the semiconductor material comprising the phase modulator contained in each waveguide of the central region of the arrayed waveguide grating device. Carrier injection into these InP based waveguides can achieve relatively large $(\sim 1 \%)$ modulation of the index in a short time $(\sim 1$ nsec). Alternately, reverse biasing can achieve moderate $(\sim 0.3 \%)$ modulation of the index in a very short time ( 1 psec, or limited by the device parasitics). 


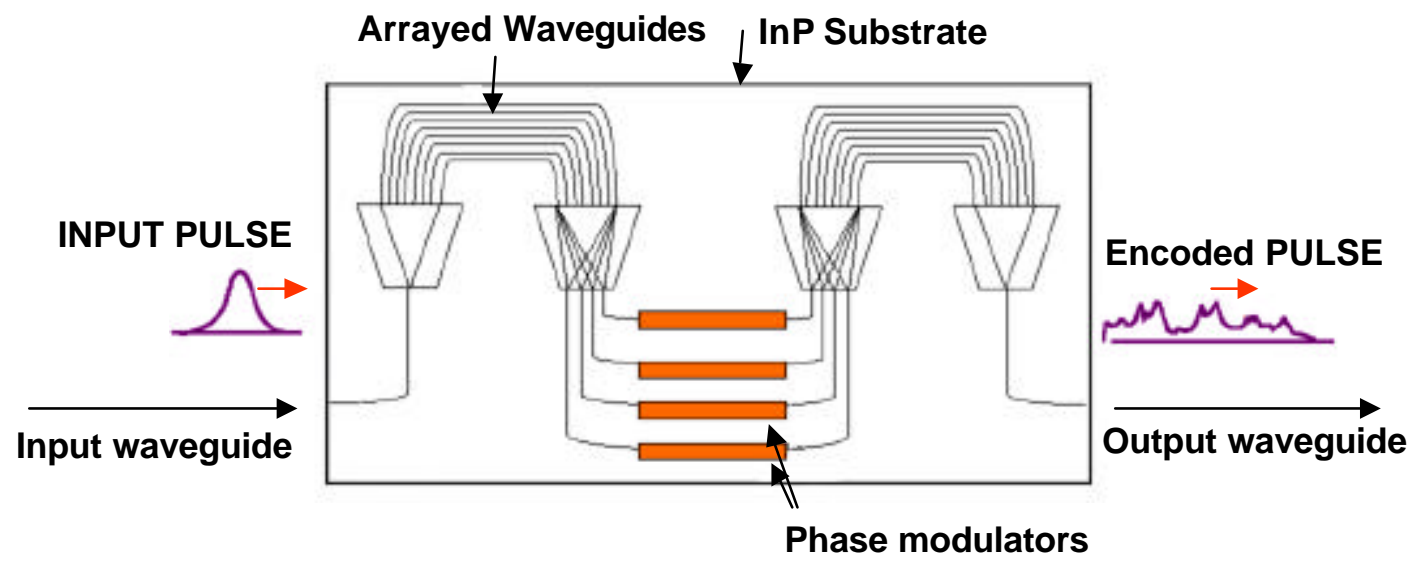

Figure 3: Arrayed Waveguide Grating (AWG) spectral phase encoder

\subsection{Accomplishments}

\section{Free Space Encoder Demonstration}

In FY01 a free-space femtosecond spectral phase encoder was designed and parts were procured. The design of the pulse shaper, shown in Figure 4, required the optimization of spectral (horizontal) dispersion in the grating direction and Gaussian beam optics in the lateral direction. The bandwidth of the commercial femtosecond laser and the physical dimensions of the spatial light modulator (SLM) at the Fourier plane fixed the linear dispersion required for the free space design. A typical spectral measurement of a compressed Pritel ultrafast optical clock pulse for 300fs pulses is shown in Figure 5. To pass $90 \%$ of the pulse power, the bandwidth designed to pass through the SLM was fixed at $13.8 \mathrm{~nm}$. The SLM consists of 128 pixels with $100 \mu \mathrm{m}$ width and $200 \mu \mathrm{m}$ height. The linear dispersion of the system was calculated to be 0.92 $\mathrm{mm} / \mathrm{nm}$ by using:

$$
\frac{\mathrm{mm}}{\mathrm{nm}}=\frac{\partial l}{\partial \lambda}=\frac{f m}{d \cos \beta}
$$

where $\partial l$ is the SLM length $(12.8 \mathrm{~mm}), \partial \lambda$ is the bandwidth of the laser pulse to transmit, $m$ is the diffraction order of the grating, $f$ is the focal length of the lens, and , $d$ is the period of the grating. A summary of the important design values is shown in Table 1. 


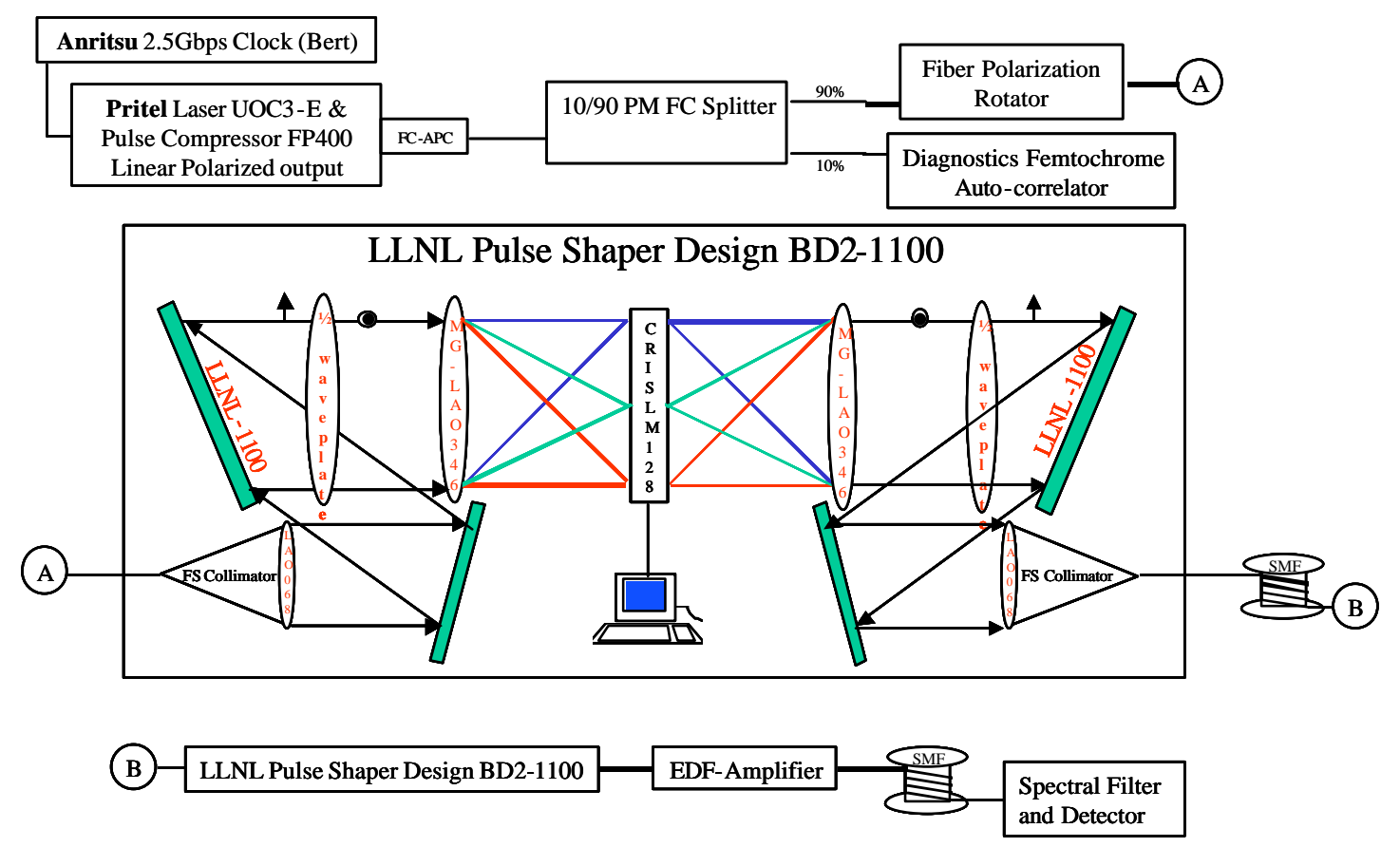

Figure 4: Diagram of free space demonstration system under construction

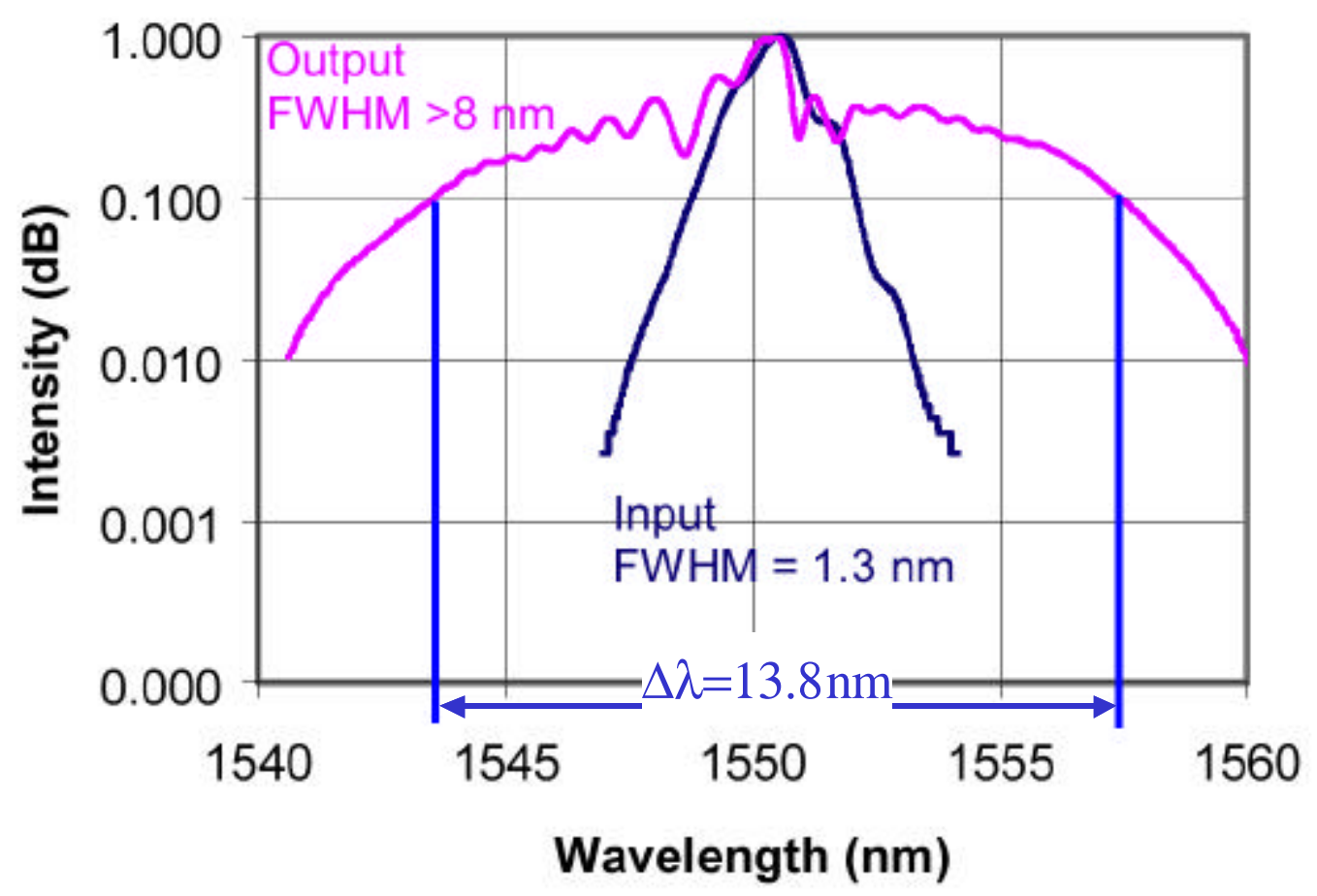

Figure 5: Pritel Laser spectral bandwidth. Indicated $\Delta \lambda$ for the $300 \mathrm{fs}$ pulse was chosen for $90 \%$ power throughput. 
Table 1: Pulse Shaper Design Summary

\begin{tabular}{|l|c|c|}
\hline Description of Variable & Amount & Units \\
\hline SLM $1 / 2$ height & 6.35 & $\mathrm{~mm}$ \\
\hline Incident spot size (collimation) & 14.6 & $\mathrm{~mm}$ \\
\hline Grating frequency & 1100 & $\mathrm{I} / \mathrm{mm}$ \\
\hline Diffraction order & -1 & \\
\hline Incident angle & 64.59 & Degrees \\
\hline Average Beta & -53.3 & Degrees \\
\hline Center Wavelength & 1550 & $\mathrm{~nm}$ \\
\hline BW to pass & 13.8 & $\mathrm{~nm}$ \\
\hline f nominal & -500.0 & $\mathrm{~mm}$ \\
\hline Gaussian Beam Factor & 2.44 & \\
\hline X spot size at SLM (want <200) & 129.53 & $\mu \mathrm{m}$ \\
\hline Y spot size at SLM (want <100) & 92.99 & $\mu \mathrm{m}$ \\
\hline Grating Size (ruled direction) & 34.03 & $\mathrm{~mm}$ \\
\hline Grating Size (other dir) & 14.6 & $\mathrm{~mm}$ \\
\hline
\end{tabular}

The Gaussian beam focusing condition was used to determine the individual spectral spot diameter, $w$, for diffraction limited focusing as:

$$
w=2.44 \frac{f \lambda}{D}
$$

where the focal length of the lens, $f$, and the incident spot diameter, $D$ were chosen to avoid overfilling the SLM pixels for a fixed wavelength $\lambda$. The design was achieved by optimizing the grating dispersion and Gaussian focusing condition by iterating the focal length of the lens and the period of the diffraction grating using commercially available, standard values. Table 1, summarizes the design completed during FY01. These design parameters were used to develop a "real world" system design incorporating commercially available lenses and gratings by using the commercial ray tracing package ZEMAX. This system was set-up, aligned and demonstrated in FY02. A ray trace showing the collimation lens, diffraction grating and focus at the Fourier plane of the three wavelengths traced are shown in Figure 6.

In addition, in FY01 the Pritel femtosecond erbium doped fiber laser was received and characterized (FY01-FY02), results are shown in Figure 7 and Figure 8. For instance, pulsewidth measurements of the picosecond laser pulses and the compressed femtosecond pulses were made using a scanning autocorrelator. The dispersion-free autocorrelator measures the correlation (through second harmonic generation) of the pulse and a time shifted replica of the same pulse. The pulse width of the laser, $1.9 \mathrm{ps}$, and the compressed pulse, 300fs, were determined by dividing the measured FWHM, shown in Figure 7, by $50 \mu \mathrm{s} / \mathrm{ps}$, a factor determined by the speed of the rotating mirror in the autocorrelator and the sech pulse shape. Figure 8 shows the spectral measurements, made using an optical spectrum analyzer 

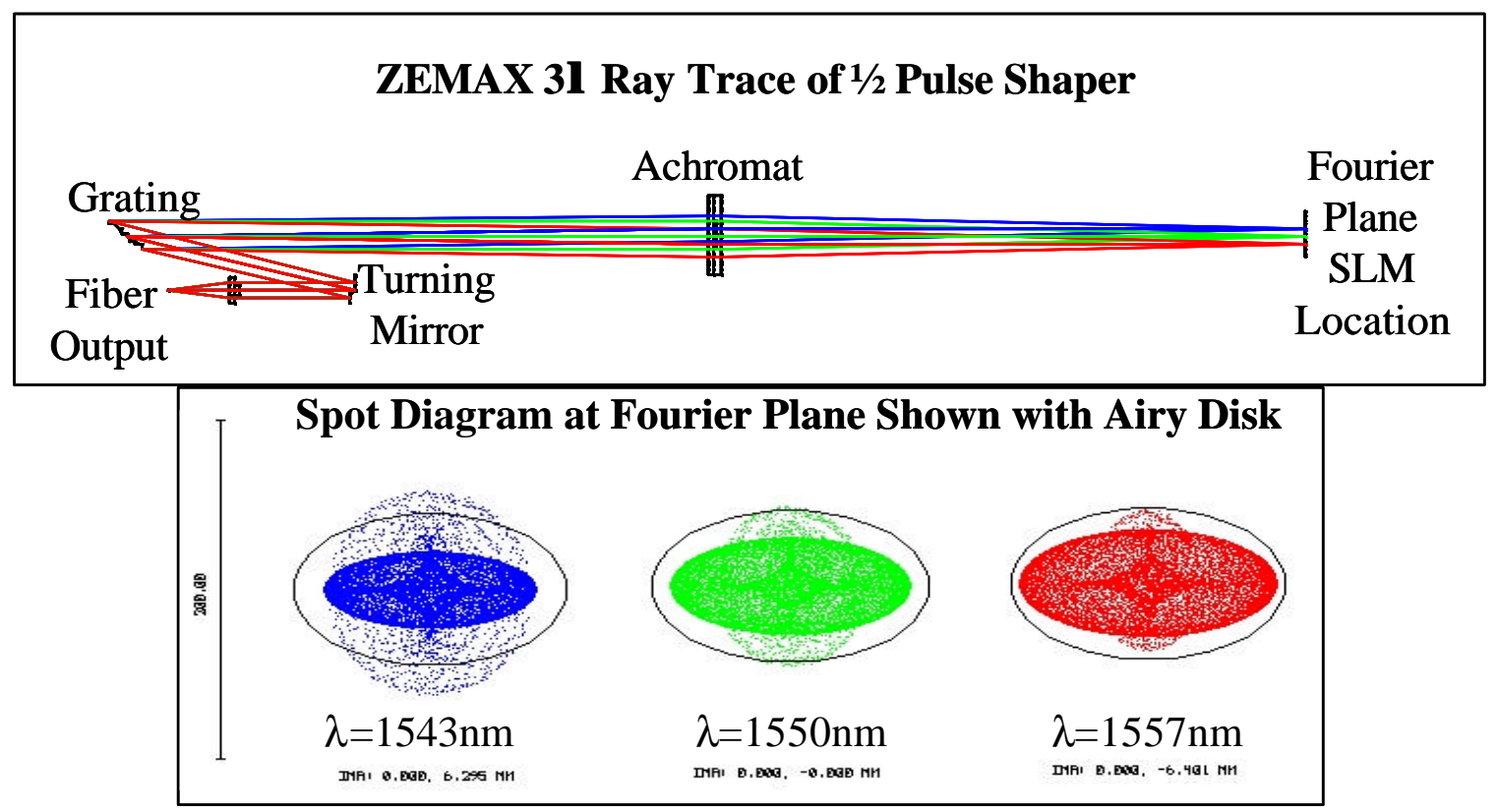

Figure 6: ZEMAX Ray Tracing Results for $1 / 2$ of the Pulse Shaper. The other $1 / 2$ of the pulse shaper is the mirror image at the Fourier Plane/SLM Location.

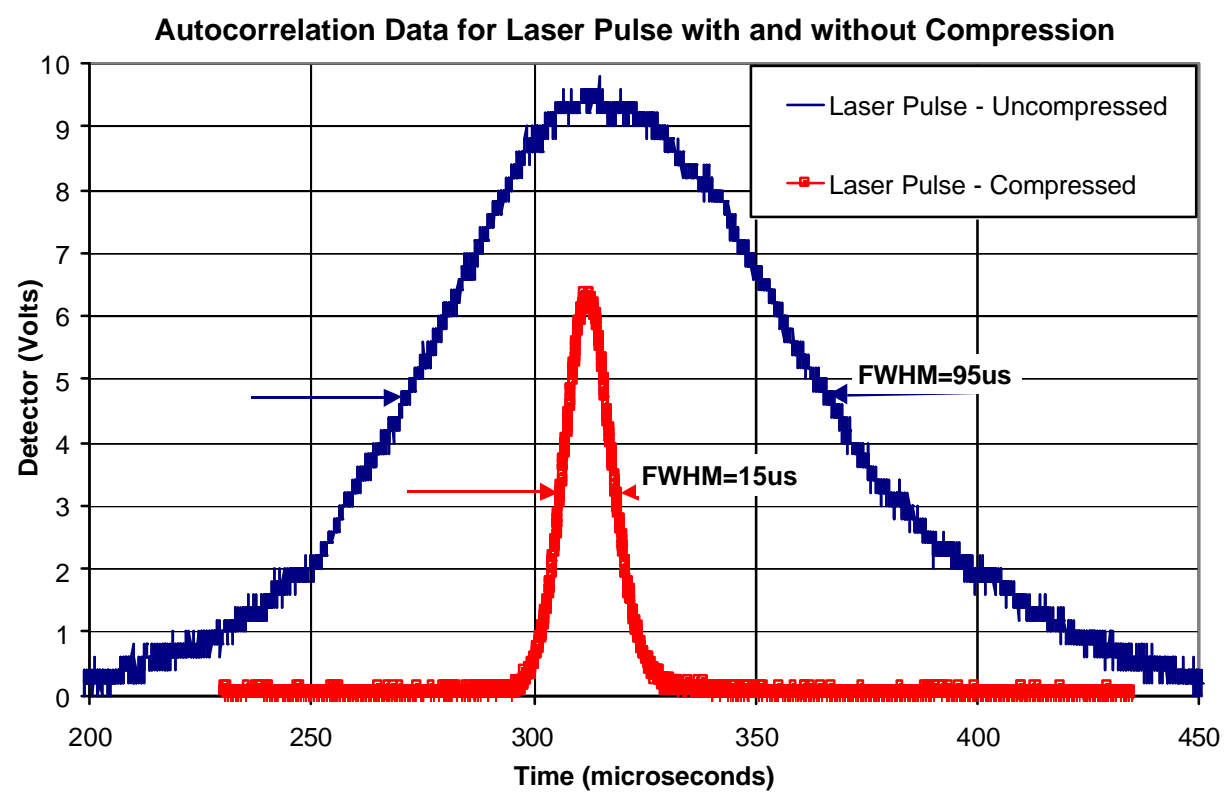

Figure 7: Pulse width measurements of the Laser and Compressor Outputs. 95 $\mu$ s laser pulses correspond to $1.9 \mathrm{ps}, 15 \mu \mathrm{s}$ compressed laser pulses correspond to $300 \mathrm{fs}$ 


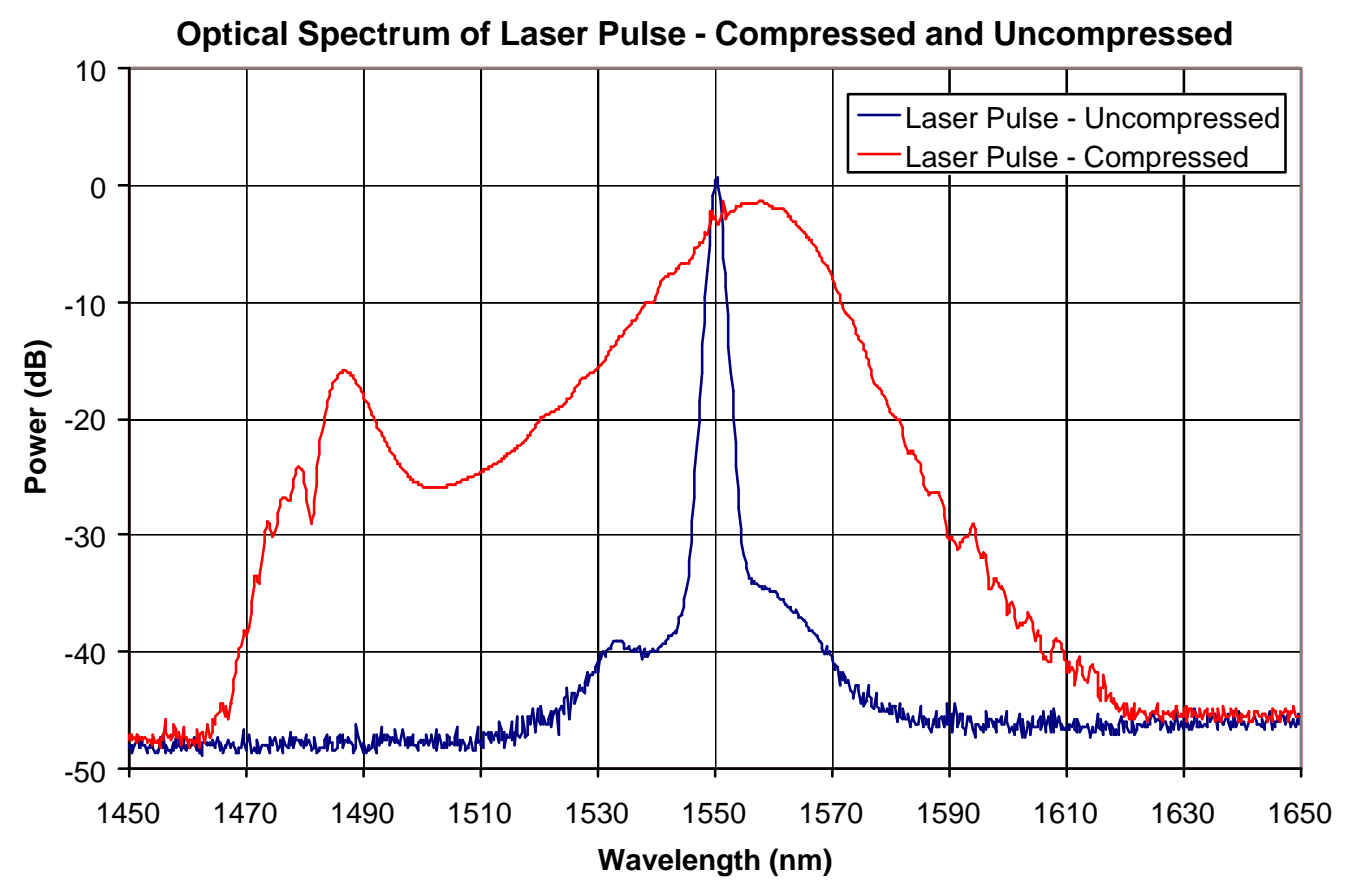

Figure 8: Spectral measurements of the Laser and Compressor Outputs

\section{FY02 - Transisition to Integrated Device Research}

In FY02, UC Davis in collaboration with LLNL submitted and was funded for a DARPA program entitled "Ultra-Compact, Rapidly Reconfigurable, Monolithic Optical-Code Division Multiplex Access Technology for Next Generation Communication Networks." The focus of this joint effort was on increasing the spectral efficiency (bits/Hz) of communications networks. In this program, the methods of O-CDMA are being applied to multiple-user access, different than the secure technology developed during FY01 and FY02 at LLNL. As the LLNL portion of the DARPA program was focused on device development and integration, the focus of the LDRD shifted toward device development in FY03. The team at UCD assumed responsibility for modeling of fiber propagation and table top demonstrations. Below we highlight some of the relevant results from this joint activity (encoder demonstration and system modeling).

\section{Free Space (Table Top) Encoder Demonstration}

The pulse shaper design and hardware discussed above were transferred to UC Davis for experimental validation of spectral phase encoded O-CDMA. The methods of encoding were changed to include multi- user access of a fiber optic transmission media and additional encoding hardware was developed and integrated into the system designed above to demonstrate encoding with multi-access interference. The basic system diagram is shown below in Figure 9. 


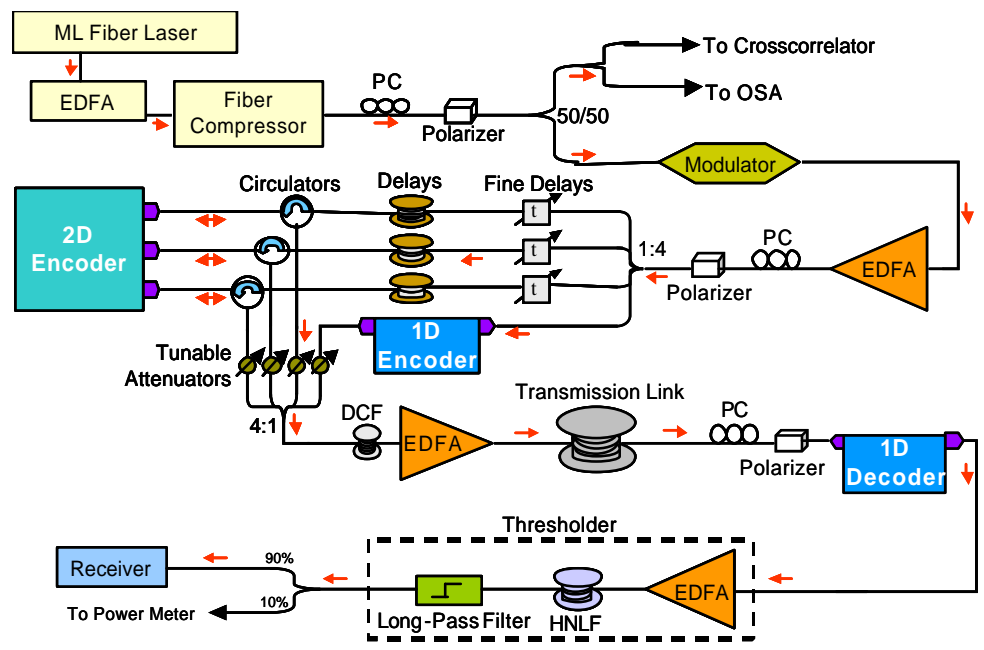

Figure 9: Darpa Free Space System Diagram

The $1 \mathrm{D}$ encoder of the figure above is the same as that designed and shown in Figure 4. The remaining pieces of Figure 9 include multi-access interference users optically combined into the transport fiber. The 1D encoder is shown in Figure 10 below. The entire system was used to demonstrate the encoding and decoding of a principal user (using the $1 \mathrm{D}$ encoder) while 3 additional user codes were operating simultaneously (using the 2D encoder).

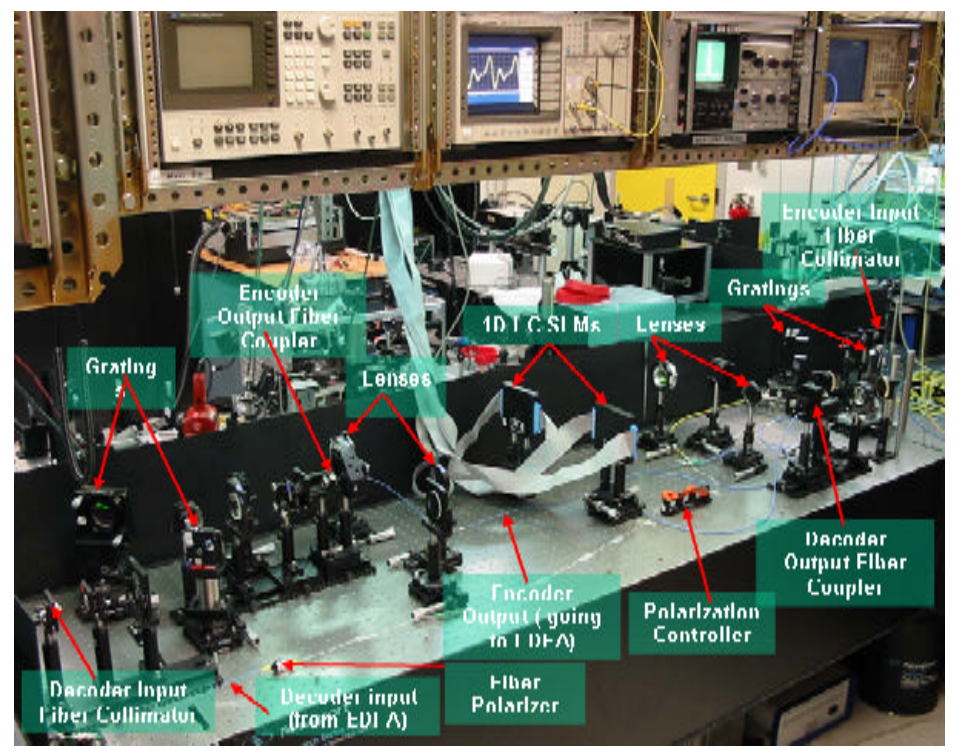

Figure 10: 1D SLM based Free Space Encoder and Decoder

The $1 \mathrm{D}$ encoder was used to encode the optical carrier for user 1 while the $2 \mathrm{D}$ encoder was used to encode the interferers. The decoder setup was used to do the autocorrelation of all 4 signals and the recovered temporal plots of Figure 11 show that only the user 1 pulse passed through the decoder with sufficient amplitude to be detected. The interferers, which did not have proper decoding, passed through as noise. 
Superposition of Four Users

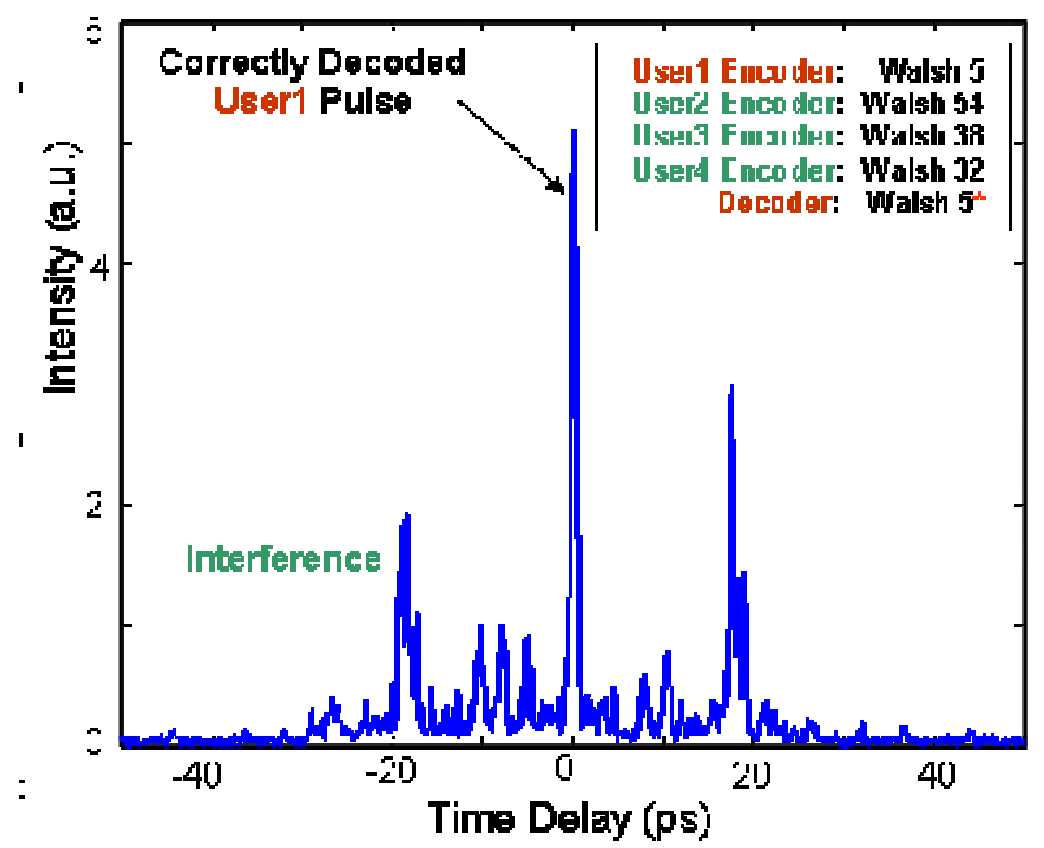

Figure 11: Properly decoded pulse with 3 interferers present

Security of encoded pulses is increased as the number of encoding bits (and the number of states for each bit) increases. Measurements shown below in Figure 12 show the effects of increasing the number of encoding bits which demonstrate increased temporal spreading and decreased amplitude possible using larger codes.

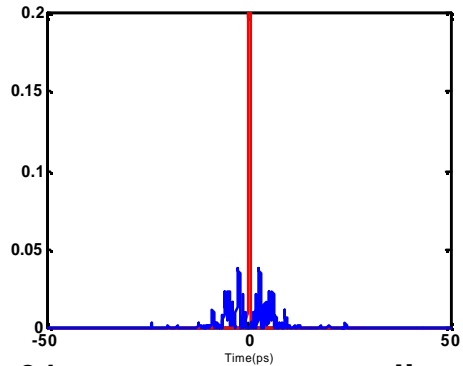

$31 \mathrm{~m}$-sequence encoding produces 25 psec spread

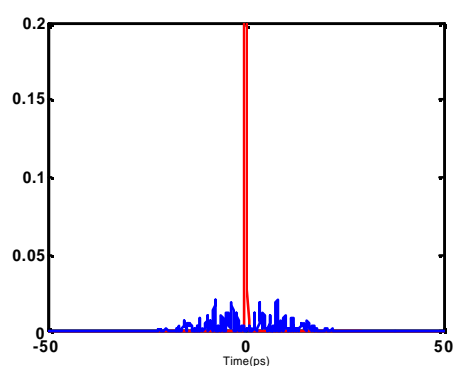

$63 \mathrm{~m}$-sequence encoding produces 46 psec spread

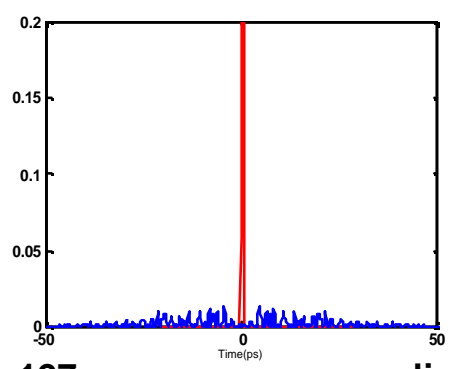

127 m-sequence encoding produces 84 psec spread

Figure 12: Pulse spreading increases with number of bits of encoding

Another key concept, that of a thresholding detector, originally proposed in FY01 but dropped at LLNL due to funding cuts, has been demonstrated (at UC Davis) with DARPA funding. The problem in detection of O-CDMA signals is that even high speed optical receivers cannot discriminate between an incorrectly decoded pulse and a correctly decoded pulse. The energy content of each of the two pulses is the same and is averaged due to the slow response of a typical optical receiver. The concept originally proposed, and demonstrated by [5] utilizes self 
phase modulation (SPM) of the high peak power in a properly decoded data stream. SPM causes spectral broadening of the pulse that can be filtered at the optical receiver.

The DARPA O-CDMA testbed incorporates 500 meters of highly non- linear fiber which allows sufficient spectral broadening. Figure 13 shows the operation of the nonlinear thresholder. Self-phase modulation causes the power from the central peak to shift to higher and lower wavelengths, with significant power concentrated between $1560 \mathrm{~nm}$ and $1580 \mathrm{~nm}$. A longpass filter with a $1568 \mathrm{~nm}$ cutoff wavelength allows the longer wavelength components to pass through to the detector, and the total power for the correctly decoded pulse shows at least a 23 $\mathrm{dB}$ contrast ratio when compared to the incorrectly decoded pulse.

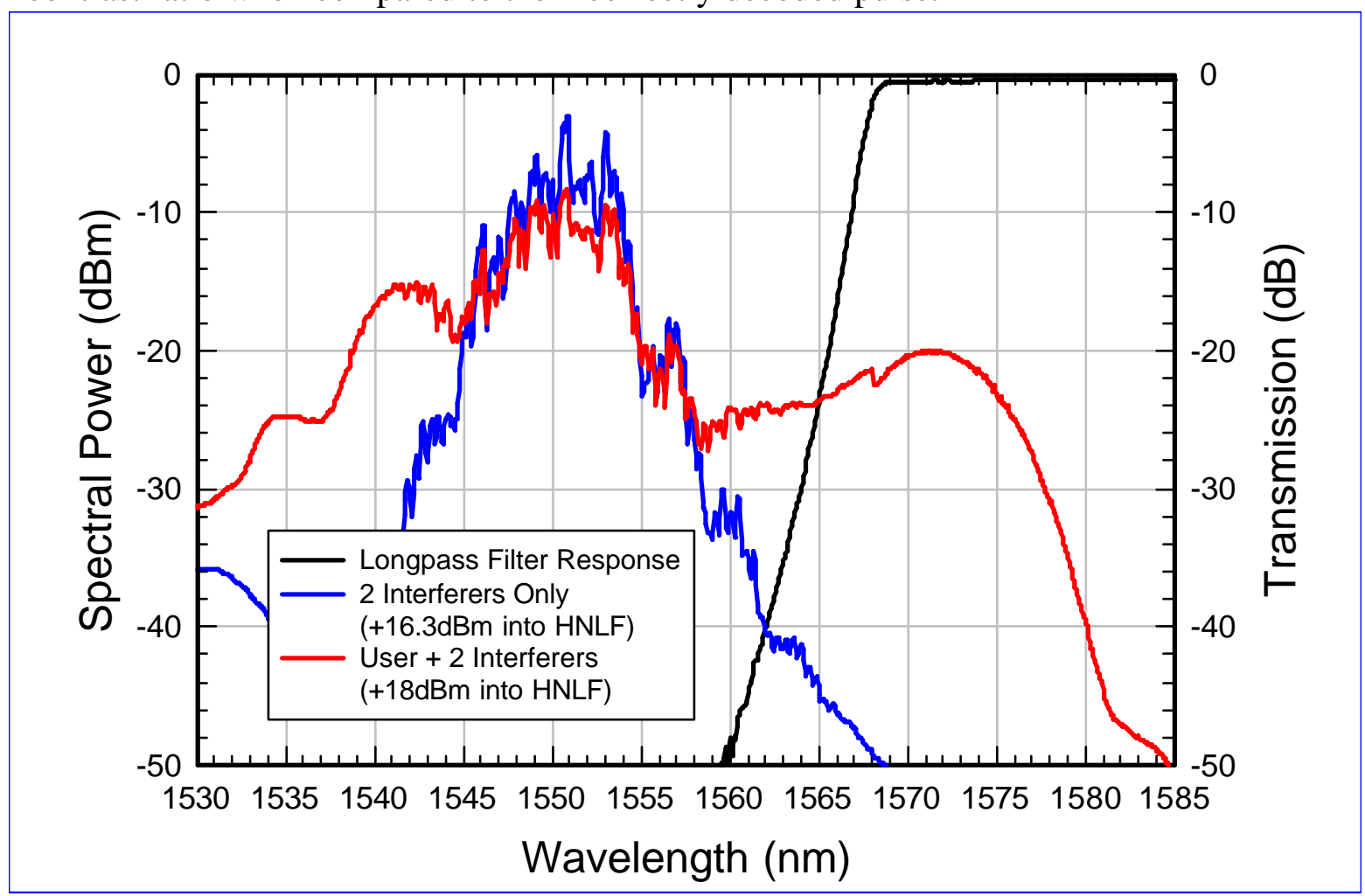

Figure 13: Non Linear Thresholder spectral response. The red trace shows the effects of Self Phase Modulation which allows detection of properly decoded O-CDMA pulses

\section{System Modeling}

Physical layer modeling of pulse propagation across fiber has been completed using Rsoft's Linksim tools which was purchased in FY01 for this project. Originally, the encoder and decoder were modeled using built in AWGs and phase modulators in the Linksim package. A problem was discovered with this method due to sampling errors in the modeling code. A solution was devised where MATLAB code was used to generate the spectral phase encoded pulse for propagation. Sample data from Linksim are shown below in Figure 14. 


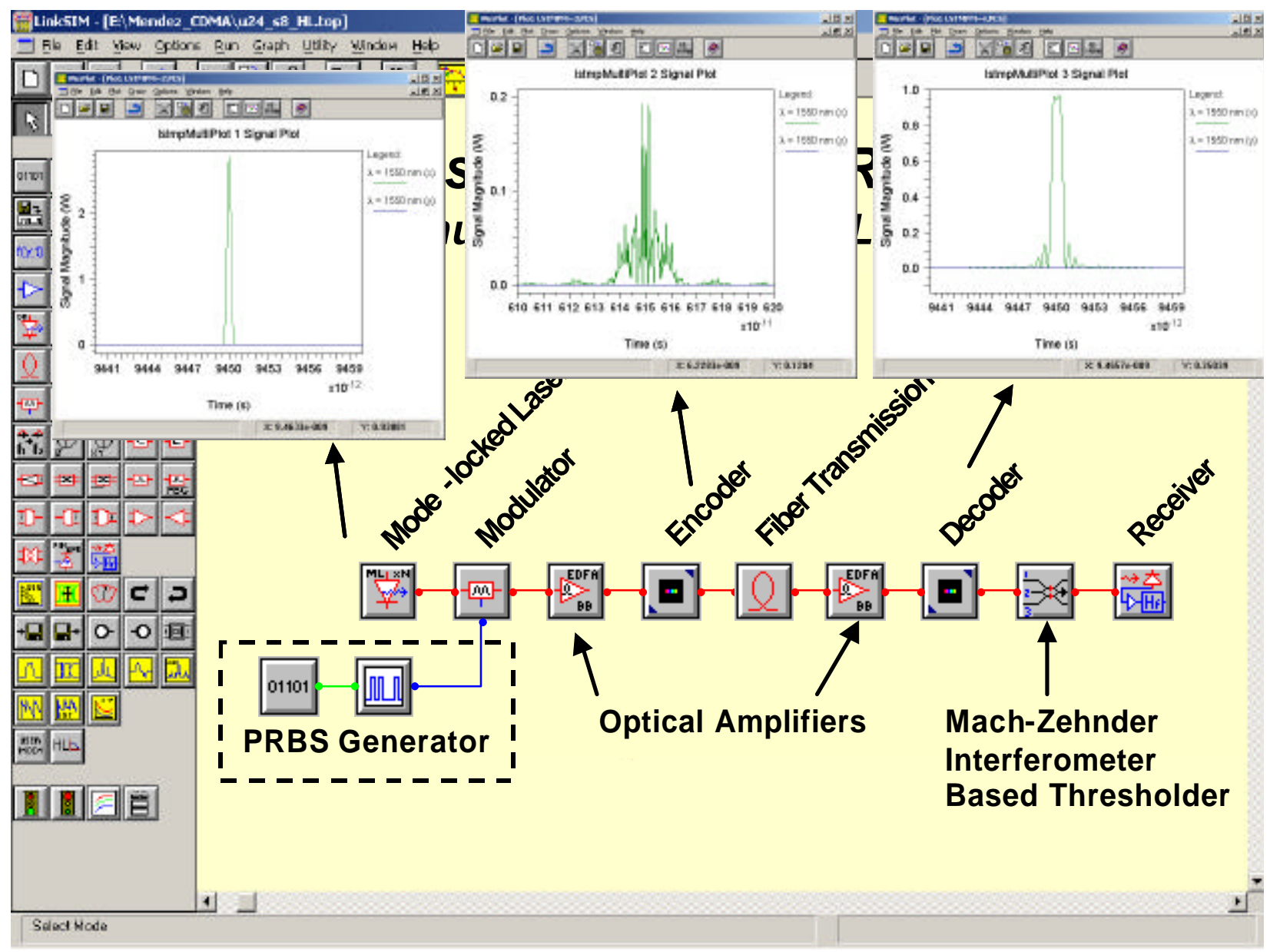

Figure 14: Linksim model showing the original, encoded, and decoded pulse.

\section{Monolithically Integrated Technology}

In parallel with our system efforts (FY01 and FY02) that were ongoing from the start, an investment was made in the enabling technologies to fabricate an integrated chip. The system work was completed in FY02 and at this point in the project, the focus was moved towards fabricating the building block of the encoder, which is the arrayed waveguide grating (AWG). The fabrication of an AWG relies heavily on complex semiconductor material growth and plasma etching. Figure 15 shows a monolithic AWG fabricated in the Center for Micro and Nano Technology at LLNL.

During FY01 we re-initialized the MOCVD (metal organic chemical vapor deposition) semiconductor growth facility on-site. The growth of the InP-based quaternary $\operatorname{In}_{1-x} \mathrm{Ga}_{\mathrm{x}} \mathrm{As}_{\mathrm{y}} \mathrm{P}_{1-\mathrm{y}}$ material is difficult due to the two stoichiometric material parameters $\mathrm{x}$ and $\mathrm{y}$. The advantage of this material is the freedom available in tuning the composition, bandgap and index of refraction while still maintaining lattice matching to the InP substrate. The MOCVD grown material utilizes an InGaAsP quaternary crystal layer, lattice matched to the InP substrate. The combination of two group III and two group V materials forms a two parameter set which allows tuning of the bandgap and hence index of refraction of the material while maintaining lattice 


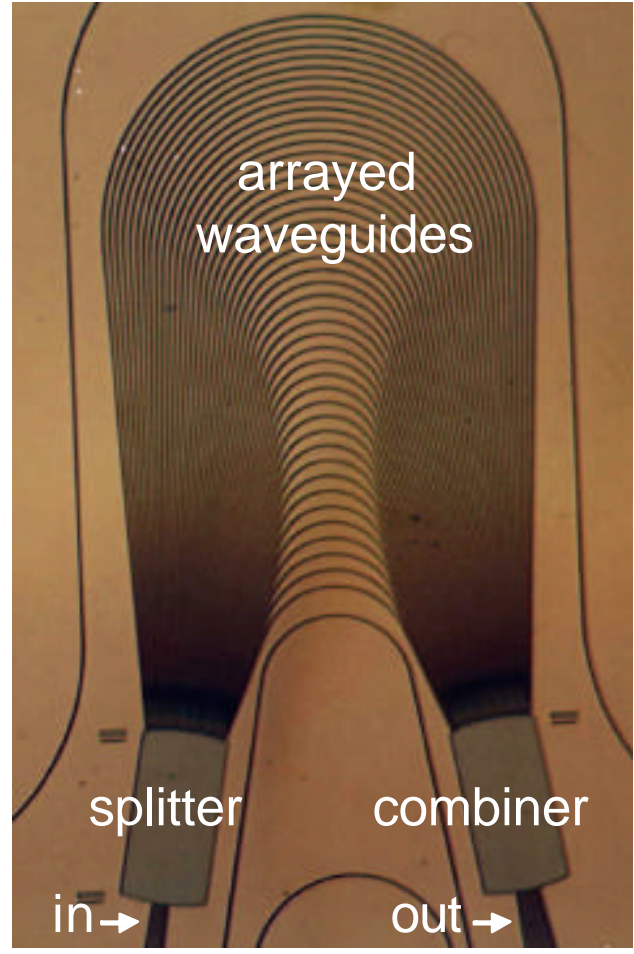

Figure 15a: Photograph of fabricated AWG device. Light with many wavelengths (channels) is multiplexed from 1 input guide to $n$ output guides by splitting, constructively interfering and combining.



Figure 15b: SEM cross section of fabricated AWG device. This slice was taken looking into the splitter.

matching to the InP substrate. The waveguide design for the AWGs consists of the high index material as the core (waveguiding region) cladded by the lower index InP material. A representative schematic is shown in Figure. There are two basic designs for the waveguide: rib and ridge waveguide. A rib waveguide (shallow etch) is one in which the etching is terminated near the top of the core region. This yields a device with low loss and have low coupling loss to an optical fiber. On the other hand, if the etching is terminated after the core region the waveguide is a ridge waveguide (deep etch) which can have higher loss due to the light scattering from the etched surface which will have some finite roughness. However, a deeply etched waveguide will generally have higher confinement and in the design we are using can be made to be polarization independent. A schematic of this structure is shown in Figure. For this project we have investigated both structures. This waveguide is the fundamental building block of the encoder/decoder device. 


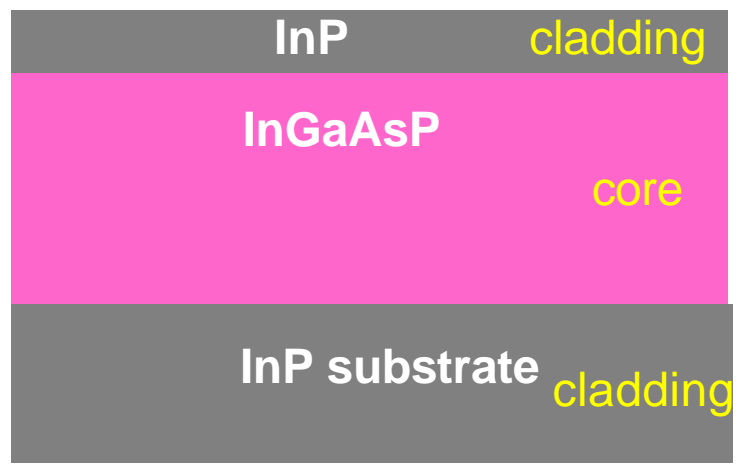

Figure 16a: Schematic of waveguide design.

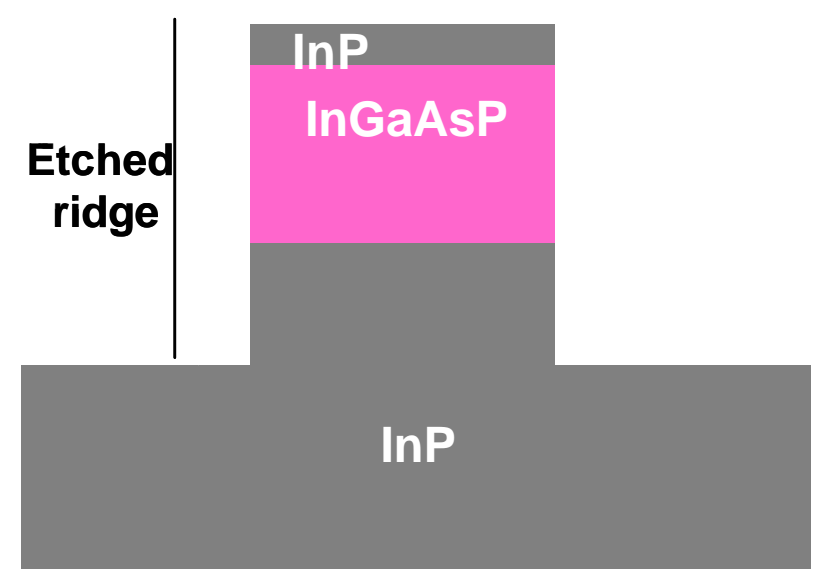

Figure 16b: Schematic of deep etched waveguide.

Initial device fabrication was performed utilizing wet etching $\left(\mathrm{HBr}+\mathrm{H}_{2} \mathrm{O}\right)$ of the waveguide regions. Figure 24: Waveguide design grown by in-house MOCVD used for AWG fabrication. 24 2shows an etched individual wave guide showing the effect of undercutting due to wet etching. The wet etching results in undesirable isotropic etching and is dependent on the crystallographic structure of semiconductors. Isotropic etching is undesirable because of the geometrical change of the waveguide ridge. For the AWG device this is problematic for the turning structure (shown in Figure 15). Furthermore, the electro-magnetic field lines will interact with the etched sidewall which nakes the smoothness critical. The optimal etching mechanism is to utilize low-damage dry-etching.

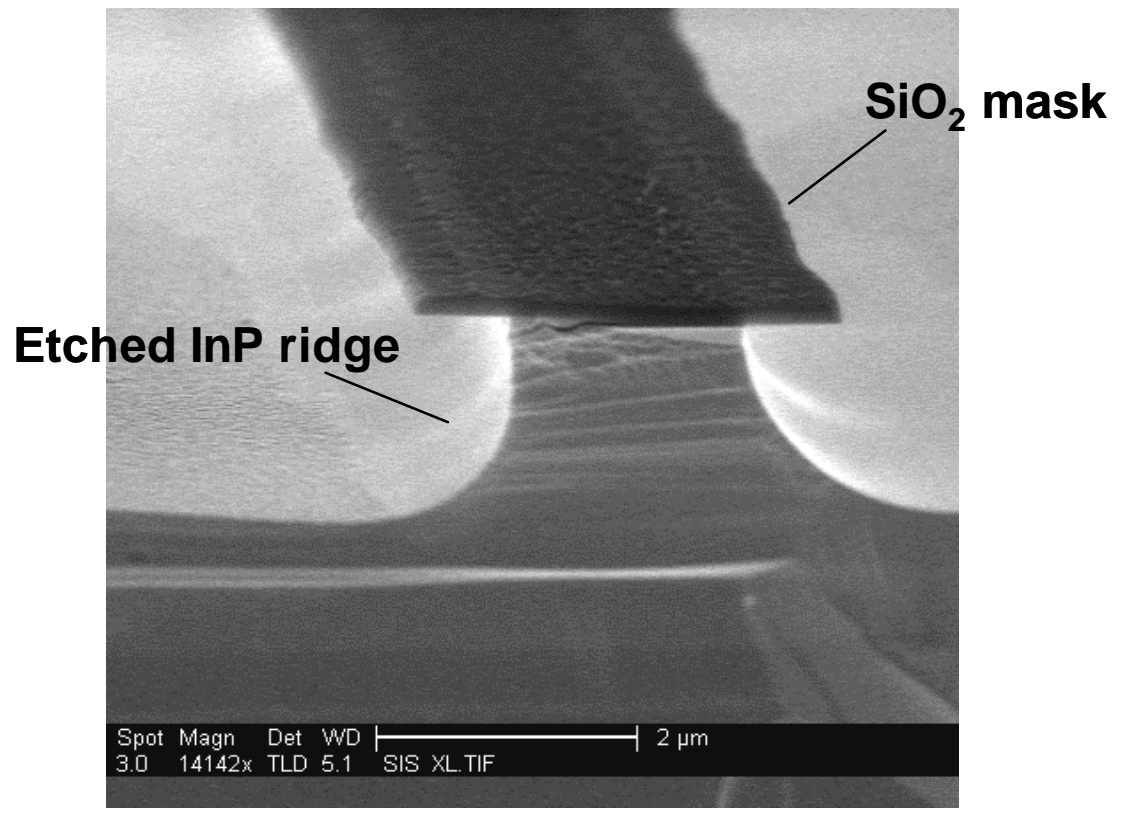

Figure 17: SEM micrographs of a wet-etched AWG, undercut due to the isotropic wet etching.

To facilitate this, we have installing an electron-cyclotron resonance (ECR) etch tool in the Center for Micro and Nano Technology. A schematic of our ECR machine is shown in Figure 
18. This system was purchased under a Crada in 1993 and was donated during FY02 to the University of California. This system was moved from B391 and installed in B153. Installation of this system was completed in 6/02. After installation, a significant amount of etch development was done in FY03, with the objective of developing a process capable of providing anisotropic etching with smooth etched surfaces.



Figure 18: of Plasma Quest Electron Cyclotron Resonance Etching (ECR) system.

In this LDRD we developed electron cyclotron resonance etching (ECR) of InP based materials using a plasma containing $\mathrm{Cb}$ and $\mathrm{H}_{2}$. This is a novel approach to etching InP based semiconductors, which has many advantages over what is currently used to etch these materials. For instance, $\mathrm{CH}_{4} / \mathrm{H}_{2}$ based dry etching of $\mathrm{InP}$ based materials is routinely done [6][7][8][9]. The drawback to this approach is the polymers that develop on the InP surface during etching, from the hydrocarbons. By carefully tuning the ratio of $\mathrm{CH}_{4}$ to $\mathrm{H}_{2}$ the amount of polymers can be reduced but typically not eliminated. Alternatively, $\mathrm{Cb} / \mathrm{Ar}$ can also be used to etch $\mathrm{InP}$ based materials. Using conventional Reactive Ion Etching (RIE) the substrate temperature is $>140{ }^{\circ} \mathrm{C}$ to have enhanced volatilization of the In compounds, such as the $\mathrm{InCl}_{\mathrm{k}}$ [10], to make a smooth etched InP surface. Devices using $\mathrm{Cb} / \mathrm{Ar}$ processesing are susceptible to "notching" at the bottom of the etched well due to the Ar sputtering and an "overcut" (positive slope from corner of the mask) due to mask erosion. 




Figure 19: Etch rate versus chlorine composition for InP and InGaAsP.

Our approach is a polymer-free means of etching InP based materials. Using a high density plasma available from the ECR source at $850 \mathrm{~W}$ we are able to run this process at $50{ }^{\circ} \mathrm{C}$ which yields flexibility in the masking material selection and should result in no device degradation compared to high-temperature plasma processing. The high ion density plasma and highly chemical etching are effective in producing etched surfaces with a smooth morphology. Figure 19 shows the etch rates for $\mathrm{InP}$ and InGaAsP using $\mathrm{Cb} / \mathrm{H}_{2}$ plasma at $1 \mathrm{mTorr}$ (ECR power $=850 \mathrm{~W}, \mathrm{RF}$ power $=250 \mathrm{~W}$ ). Below $50 \% \mathrm{Cb}$ composition the etch rates for both materials are the same. As the $\mathrm{Cl}_{2}$ is increased, the etch rate of InP becomes increased over that of InGaAsP. We found the optimal condition for etching to be with $\mathrm{Cb}=80 \%$ in terms of morphology and profile. With $\mathrm{Cb}=80 \%$ the etch rates for InP and InGaAsP are $0.81 \mu \mathrm{m} / \mathrm{min}$ and $0.56 \mu \mathrm{m} / \mathrm{min}$ respectively with a selectivity between $\mathrm{InP}$ and $\mathrm{SiO}_{2}$ of $>40$. AFM measurements were done on both the sidewall of the etched facet and the surface, as indicated in Figure 20. The sidewall rms roughness is $3.67 \mathrm{~nm}$ and the surface is $1.01 \mathrm{~nm}$. The rms roughness for unprocessed $\mathrm{InP}$ is 0.2 $\mathrm{nm}$. The surface of the etch roughness is caused by the unequal etch rates on indium and phosphorus. The sidewall roughness is primarily caused by the quality of the photolithography process, and is commonly referred to as "sidewall striations." This plasma etching is discussed in greater detail in a presentation given by R.J. Welty at The International Semiconductor Device Research Symposium, Dec. 12, 2003 [11]. 
The need for smooth etched sidewalls is particularly important for optical waveguide devices for the reduction of scattering losses. Figure 21 shows the waveguide loss versus rms roughness of the etched sidewall. The simulation was done with RSOFT BEAMPROP device modeling program. The waveguide used in this simulation has a width of 2 microns and the epilayer structure as shown in Table 2. A transmission loss of less than $5 \mathrm{~dB} / \mathrm{cm}$ is needed to realize compact and highly integrated photonic devices. A maximum sidewall roughness of $150 \AA$ is permitted for this structure. The waveguide width for this design is $2 \mu \mathrm{m}$ to maintain polarization independent operation. If the width of the waveguide was wider the stringency for the sidewall morphology would be reduced.

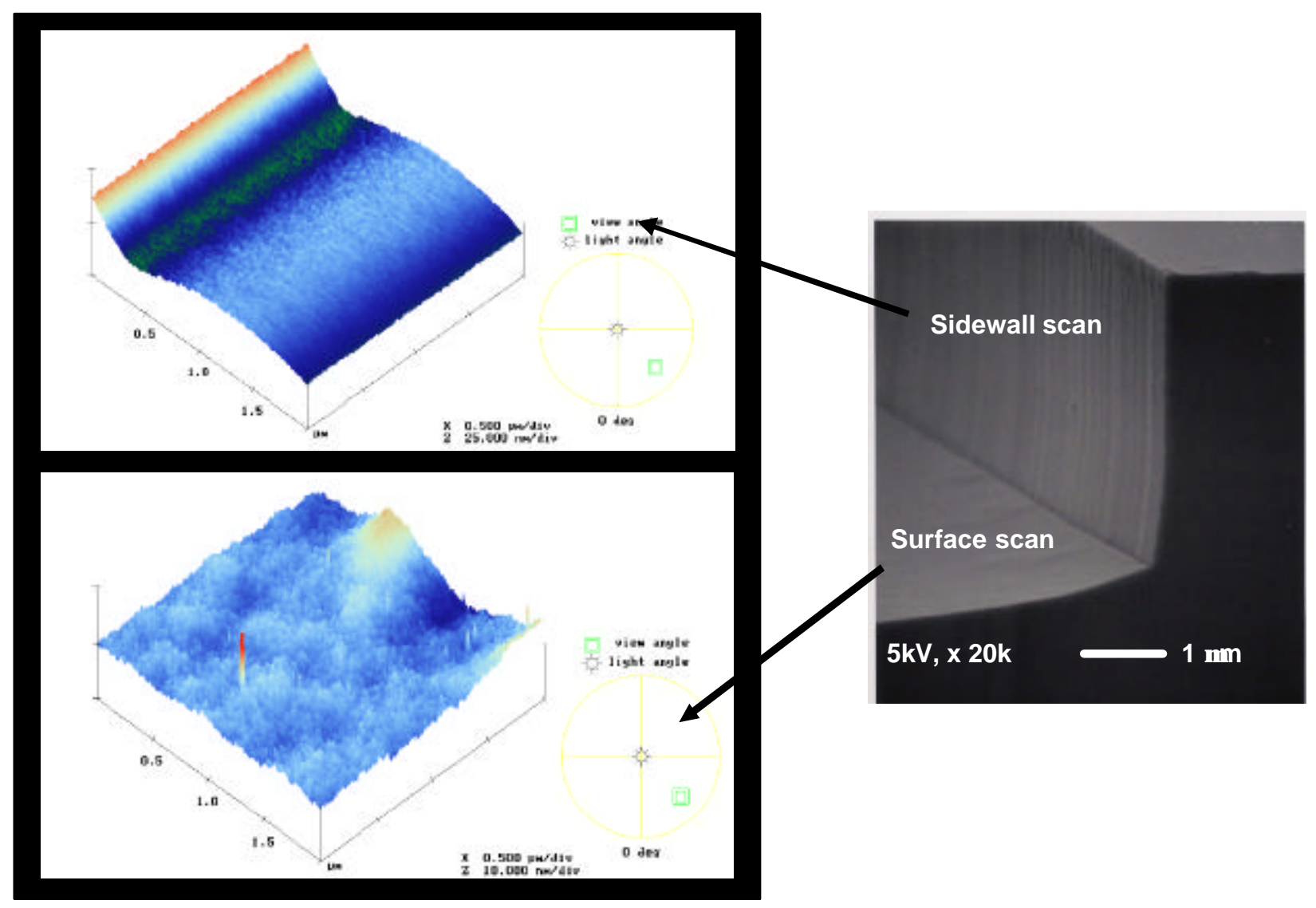

Figure 20: $\mathrm{Cl}_{2}=80 \%, \mathrm{H}_{2}=20 \%$ etched InP 3.8 microns. AFM sidewall and surface scan show very smooth morphologies of $\mathbf{r m s}($ sidewall $)=3.67 \mathrm{~nm}$ and $\operatorname{rms}($ surface $)=1.01 \mathrm{~nm}$. 


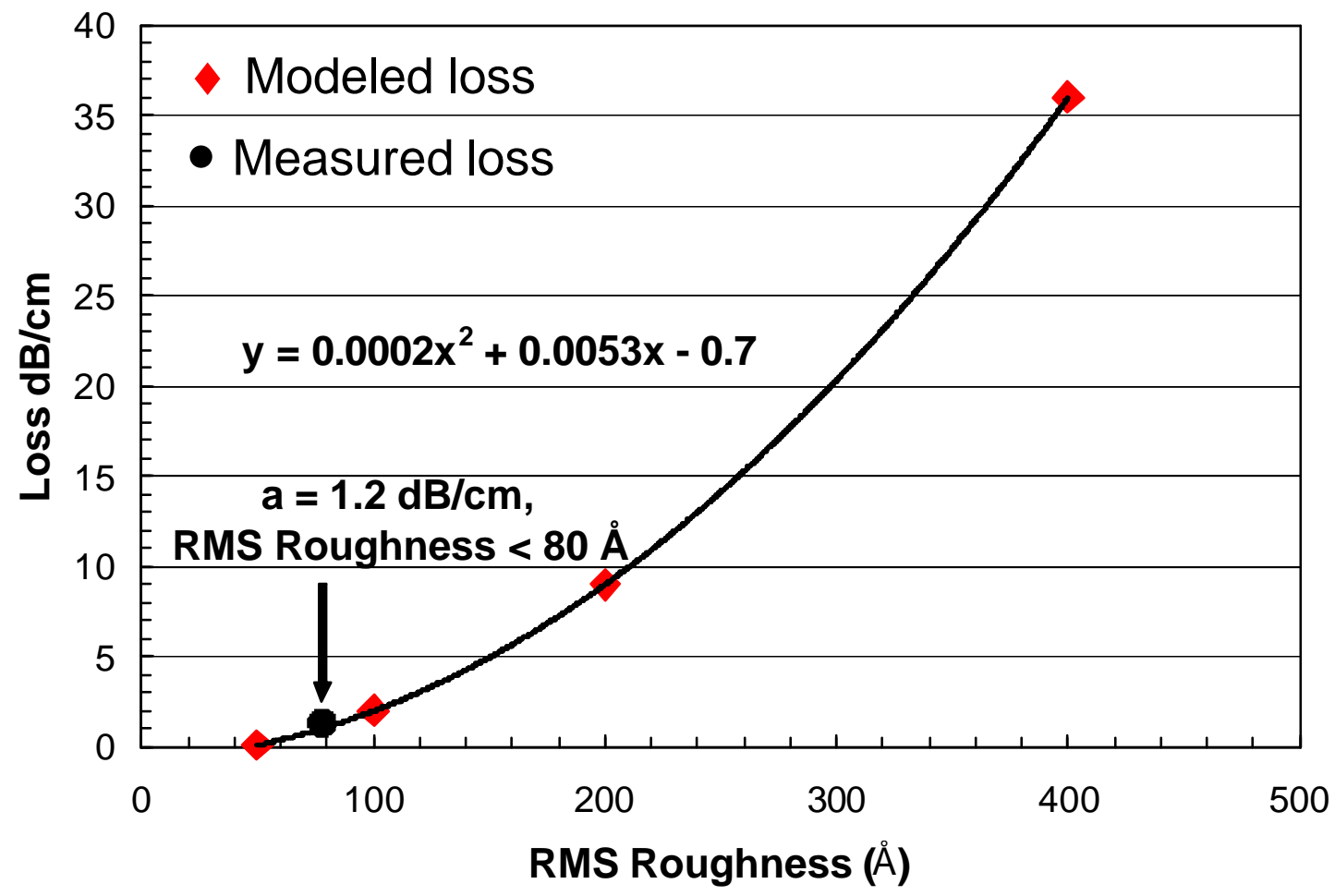

Figure 21: Modeled loss vs. rms roughness for a 2 micron wide waveguide width with etch depth of 1 micron. Waveguide structure shown in Table 2.

Table 2: Epilayer structure for ridge waveguide.

\begin{tabular}{lcccc}
\hline Layer & Material & $\mathrm{Eg}(\mathrm{eV})$ & Thickness $(\mathrm{mm})$ & Index @ $1.55 \mathrm{~mm}$ \\
\hline \hline Cladding & $\mathrm{InP}$ & 1.35 & 0.2 & 3.17 \\
Core & $\mathrm{In}_{0.77} \mathrm{Ga}_{0.23} \mathrm{As}_{0.51} \mathrm{P}_{0.49}$ & 0.99 & 0.6 & 3.37 \\
Buffer & $\mathrm{InP}$ & 1.35 & 0.2 & 3.17 \\
S.I. InP Substrate & & 1.35 & & 3.17 \\
\hline \hline
\end{tabular}

To further characterize the $\mathrm{Cb}-\mathrm{H}_{2}$ based etching, optical waveguides were fabricated and characterized. The epilayer structure for this device is shown in Table 2. The etch condition for this device is $20 \% \mathrm{H}$ and $80 \% \mathrm{Cb}$. The mask is $0.3 \mu \mathrm{m}$ of PECVD $\mathrm{SiO}_{2}$. The ECR dry etch was $1 \mu \mathrm{m}$. The depth of the etch was chosen to insure the optical mode "interacted" with the etched sidewall. BEAMPROP simulations were used in this determination. Figure 22a shows the simulated output power versus etch depth. From this simulation it is evident that the mode is tightly confined with the etch depth of at least $0.3 \mu \mathrm{m}$. Figure $22 \mathrm{~b}$ shows the optical mode profile for the 1 micron etched waveguide. 


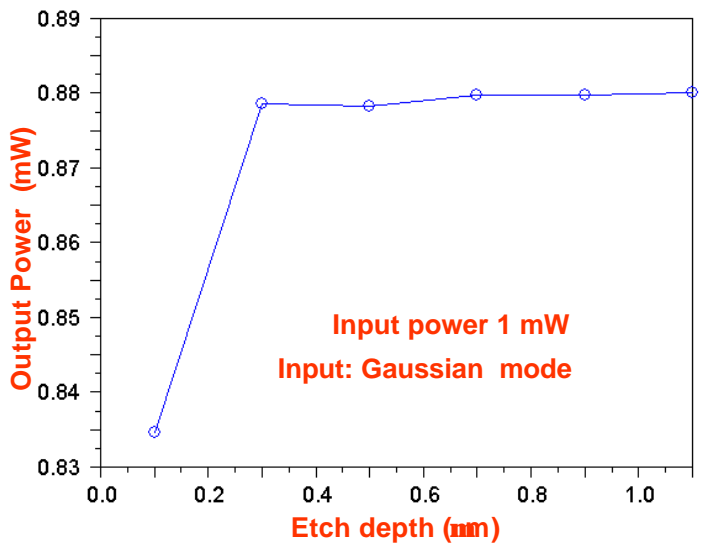

22a: Simulated output power vs. etch depth.

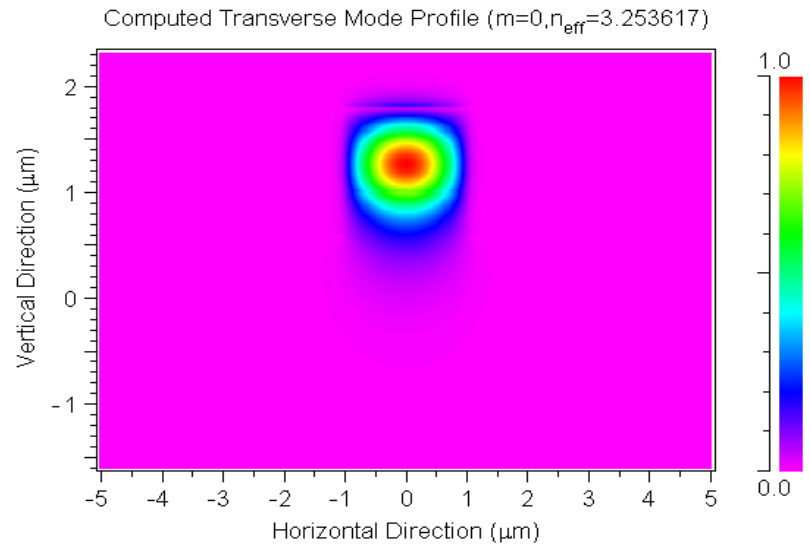

22b: Optical mode profile for etch depth of $1 \mu \mathrm{m}$.

Faby-Perot resonance measurements are done to determine the loss of the fabricated waveguides etched with ECR using mixed chlorine and hydrogen gas. The transmission loss $(a)$ of the waveguide was measured by the method of Fabry-Perot resonance [12]. The advantage of this method is that it is independent of the optical alignment and only a single measurement is needed. The waveguide can be used as a Fabry-Perot cavity and the cleaved facets act like reflecting mirrors. The electric field at the output $\left(E_{\text {out }}\right)$ of the waveguide device can be expressed as:

$$
E_{\text {out }}=\frac{\left(1-r^{2}\right) e^{-(\alpha / 2-j \beta) l}}{1-r^{2} e^{-2(\alpha / 2-j \beta) l}} E_{\text {in }}
$$

where $r$ is the is the reflectivity of the Efield at the input $\left(E_{i n}\right)$ at the waveguide facet, $l$ is the cavity length and $\beta$ is the wave vector. The ratio of the minimum and maximum optical intensity at the output of the waveguide can be expressed as:

$$
\frac{I_{\min }}{I_{\max }}=\left(\frac{1-r^{2} e^{-\alpha l}}{1+r^{2} e^{-\alpha . l}}\right)^{2} .
$$

The waveguide loss $\alpha$ then can be solved as:

$$
\alpha=\frac{1}{l} \ln \left(\frac{1}{r^{2}} \frac{\sqrt{I_{\max } / I_{\min }}-1}{\sqrt{I_{\max } / I_{\min }}+1}\right) .
$$

In order to achieve the Fabry-Perot fringe, the optical path of the cavity can be tuned by changing the length of the cavity or by changing the wavelength of the light source. The length of the waveguide is usually altered by adjusting the temperature of the waveguide. In the measurements reported here, a tunable laser is used as the light source and the wavelength of the tunable laser is swept through a certain range. For an accurate loss measurement, the line width of the laser source is required to be narrow, especially for low loss waveguides [13].

The tunable laser used for measurement is Agilent 81682 tunable laser module, with an effective linewidth is $4 \times 10^{-4} \mathrm{~nm}$ and minimum tuning step of $0.0001 \mathrm{~nm}$. The detector is Agilent 
81634 detector module. The wavelength of the tunable laser is swept from $1550 \mathrm{~nm}$ to $1551 \mathrm{~nm}$ with a $0.025 \mathrm{~nm}$ step. The light is coupled in and out the waveguide from a single mode fiber through bulk optics. The focal length is selected to match the mode field diameter of a single mode fiber and that of a $2 \mu \mathrm{m}$ wide waveguide. The measured Fabry-Perot fringe is shown in Figure 23. With the facet reflectivity of $32 \%$ for InP and the length of the waveguide is $2.5 \mathrm{~mm}$, the resulting waveguide loss is $1.2 \mathrm{~dB} / \mathrm{cm}$. Comparing this loss from the graph in Figure 21, we can infer the sidewall roughness is less than $80 \AA$ for this waveguide. We can assume the loss is due to the sidewall roughness, because of negligible absorption $\lambda_{\text {signal }} 1.55 \mu \mathrm{m}>1.26 \mu \mathrm{m}$ $\left.\lambda_{\text {material }}\right)$ and negligible loss due to scattering from the epilayer bulk and interfaces (undoped material). This was our best loss result obtained. Depending on the material quality, photolithography process, and etch repeatability our transmission loss has ranged between 1-7 $\mathrm{dB} / \mathrm{cm}$. We continue to optimize all of the variables to develop a technology capable of implementing a fabricated encoding/decoding device.

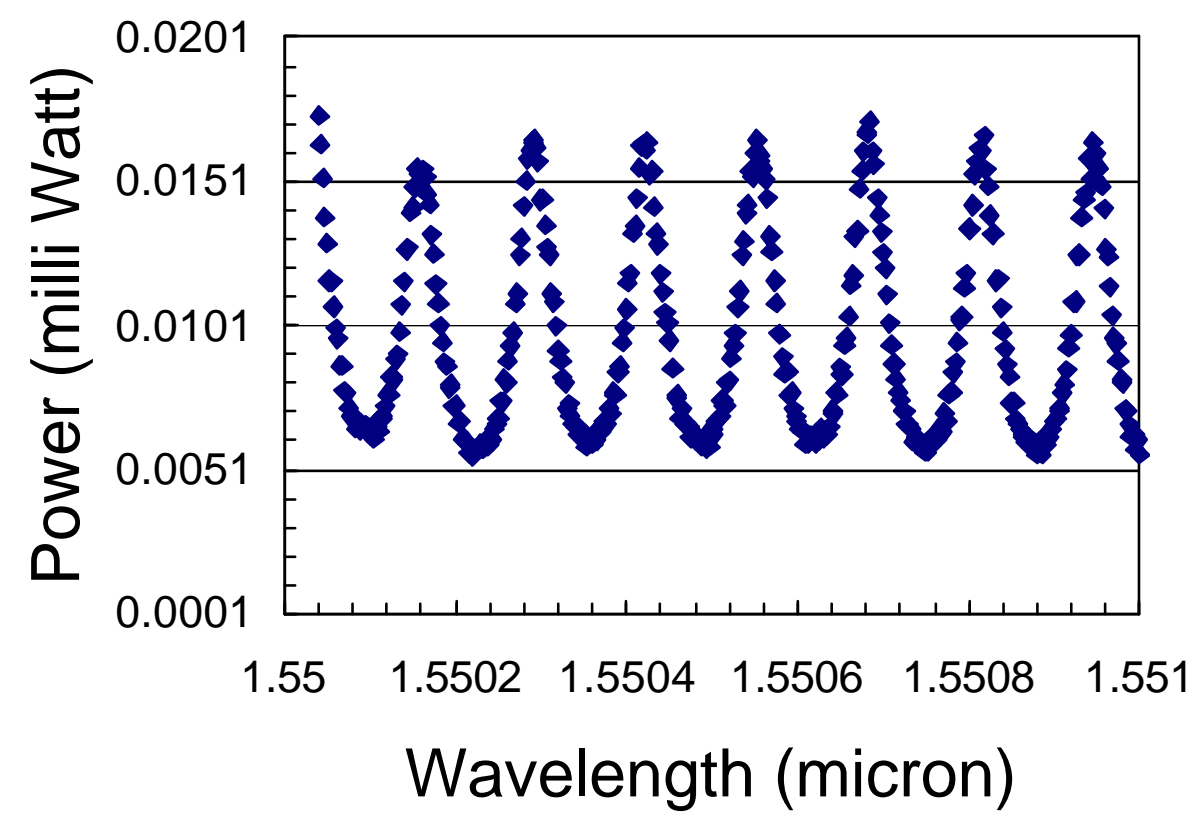

Figure 23: Fabry-Perot fringes for determination of transmission loss for fabricated optical waveguides.

The process development needed to fabricate low-loss straight waveguides took the better part of FY03. The process development that was done is the enabling technology to fabricate the AWG. With these technologies in place an integrated AWG could finally be fabricated. Inhouse material was grown using MOCVD. The AWG Epitaxial layer stack is shown in Figure 24. This design has been modified from that shown in Table 2 . The primary difference is in the second the design the top cladding layer is increased to provide better optical confinement. The bandgap of the core region is also slightly increased. 


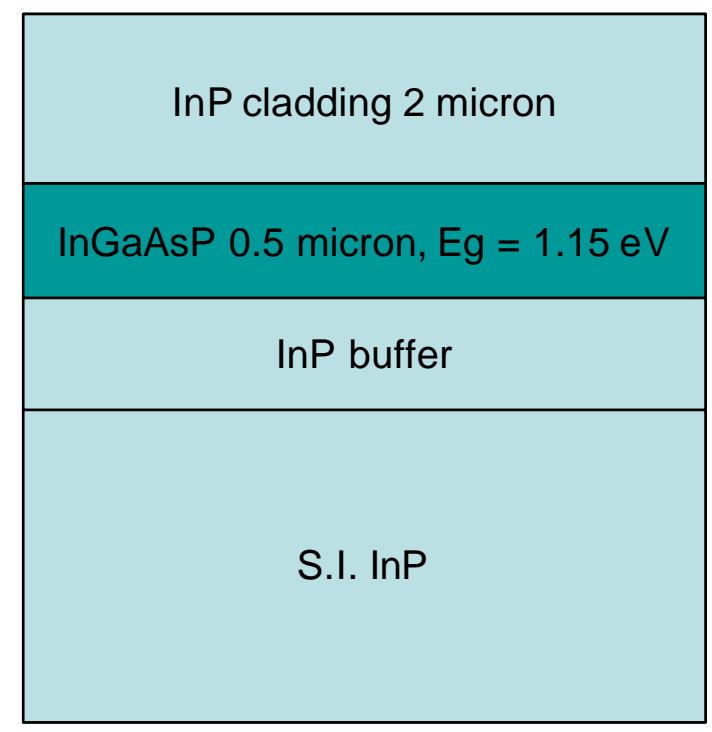

Figure 24: Waveguide design grown by in-house MOCVD used for AWG fabrication.

As shown in Figure 25 the photoluminescence mapping data, the median wavelength is $1153 \mathrm{~nm}$, off by $+3 \mathrm{~nm}$ with respect to the designed wavelength, $1150 \mathrm{~nm}$. The concentric variation seen on the PL mapping, reflecting the variations in InGaAsP composition, is associated with the characteristics of gas decomposition dynamics in the MOCVD reactor. X-ray diffraction rocking curve data is shown in Figure 26 and indicates that the lattice mismatch of InGaAsP with respect to InP is -140 arcsecond, corresponding to $0.11 \%$ of compressive strain. The periodic fringes seen on the negative angle side indicates that the interfaces between InGaAsP and InP are atomically sharp. The photoluminescence and the x-ray diffraction rocking curve data suggest that InGaAsP composition is $\operatorname{In}_{0.84} \mathrm{Ga}_{0.16} \mathrm{As}_{0.38} \mathrm{P}_{0.62}$.
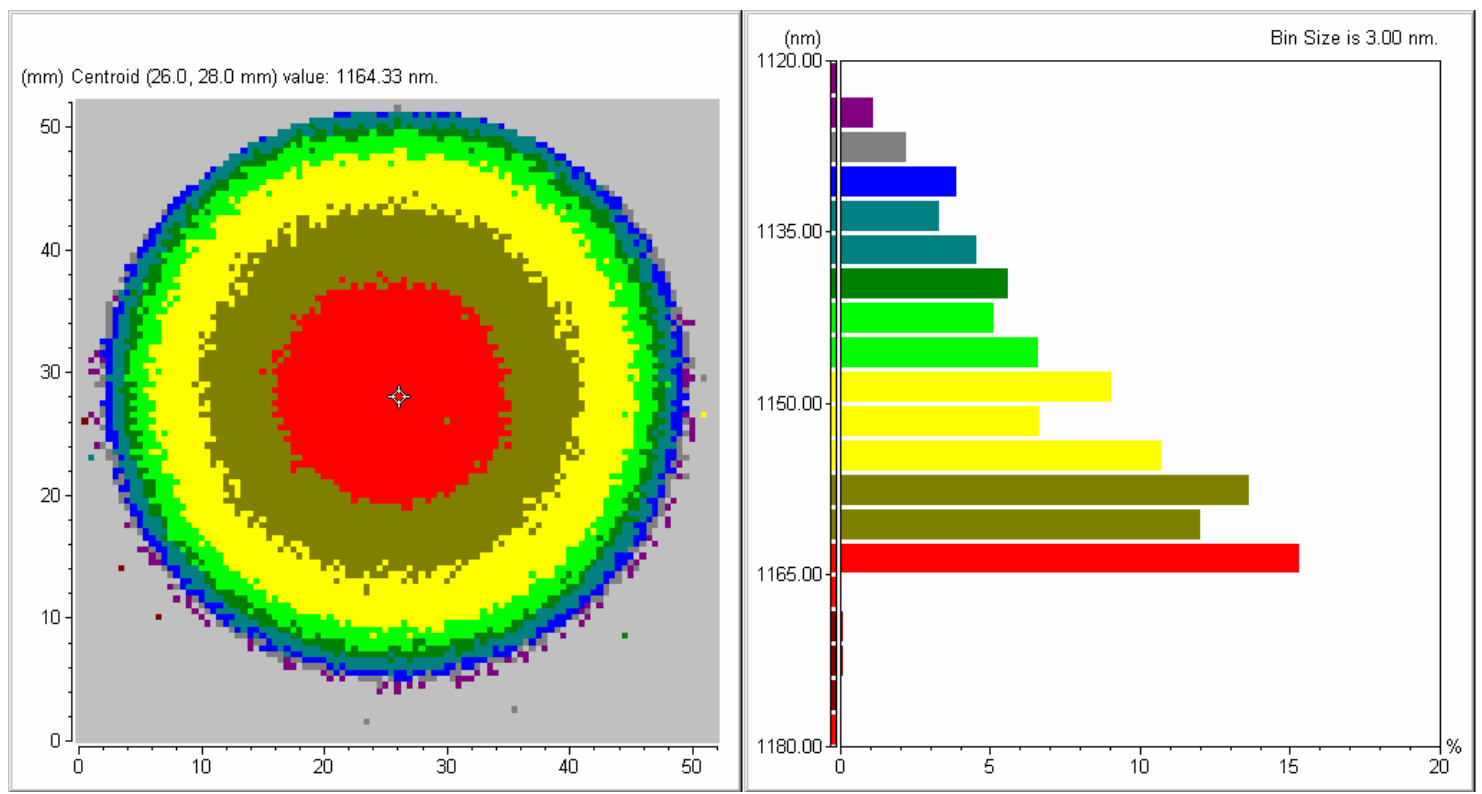

Figure 25: Photoluminescence mapping data of MOCVD grown AWG sample, SK1052. Median wavelength is $1153 \mathrm{~nm}$. The uniformity across the two inch wafer is $0.9 \%$. 


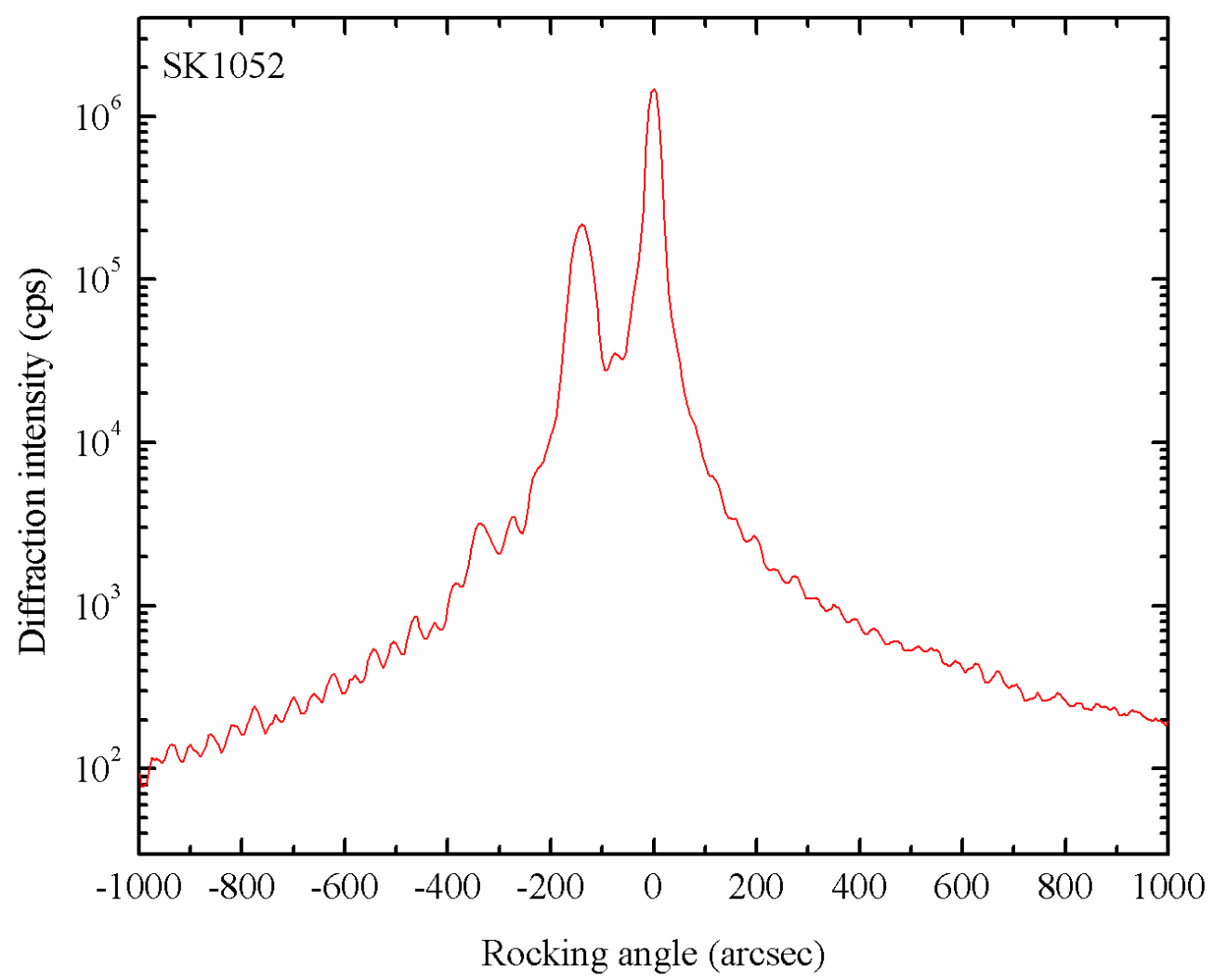

Figure 26: X-ray rocking curve of MOCVD grown AWG wafer. Peak intensity is from the InP substrate, the lower peak is for the In $0.84 \mathrm{Ga0.16As0.38P0.62}$ core region. The InGaAsP has an excellent lattice matching to the substrate with -140 arcsec $(0.11 \%$ compressive strain).

The output spectrum of a fabricated AWG is shown in Figure 27. The channel spacing is $3.4 \mathrm{~nm}$ with a signalto-noise-ration of $20 \mathrm{~dB}$. This particular device was fabricated with a shallow etch (ECR etch $80 \% \mathrm{Cl}_{2}$ and $20 \% \mathrm{H}_{2}$ ) with a depth of approximately $0.1 \mu \mathrm{m}$ etch into the core region. This was to chosen to allow a large margin for the intrinsic radiative loss due to the tight turns that this device has. Subsequent masks have been designed to allow more compact devices with deep etching (for polarization independent operation). Additionally our ECR dry etching has been continued to be improved in terms of the ability to reliably fabricate low loss waveguides. 


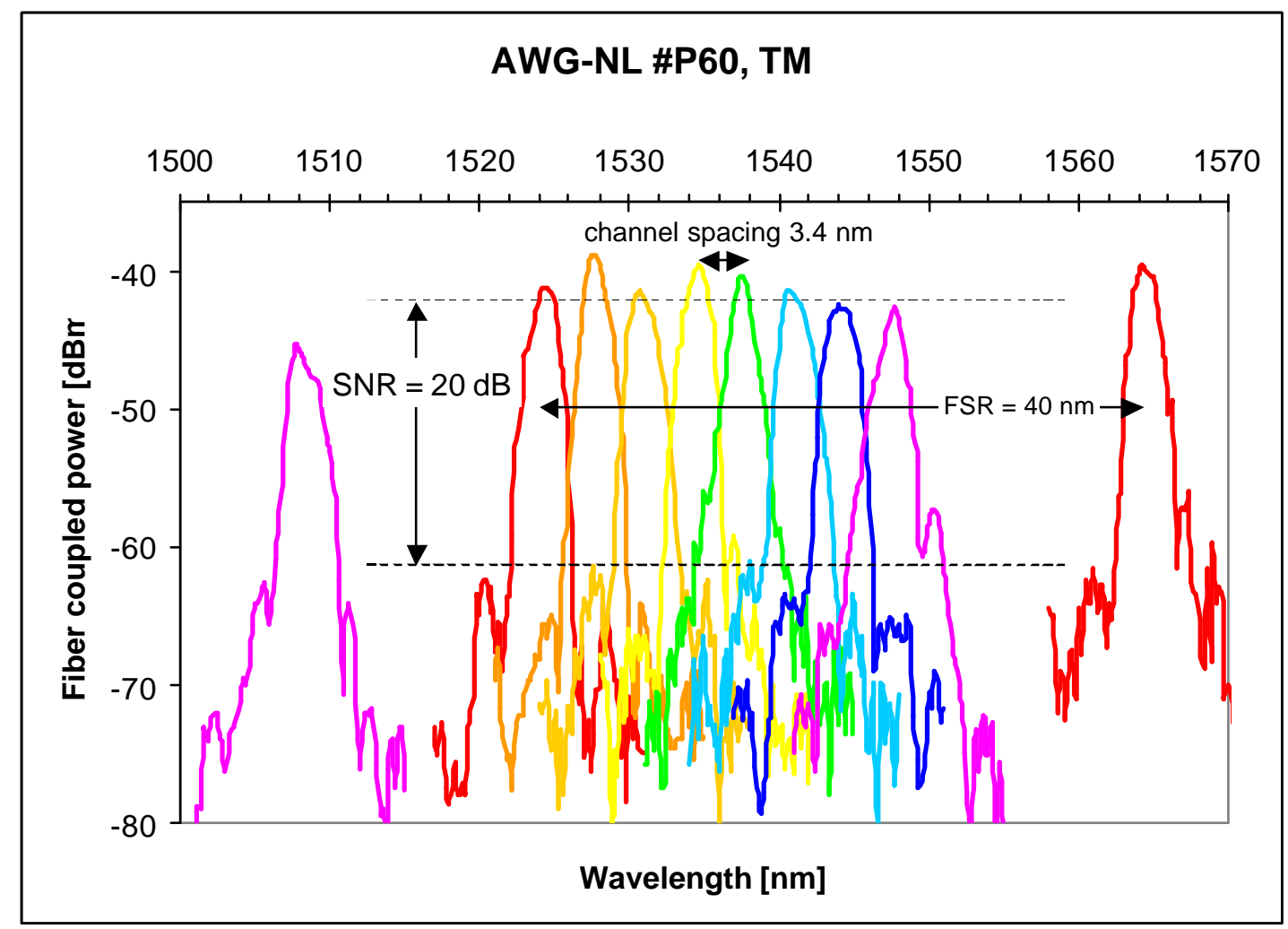

Figure 27: Output spectrum for fabricated AWG. Top view picture is shown in Figure 15.

This LDRD ended before we were able to finally integrate the modulator with the fabricated AWG. However, this work has continued as collaboration with a DARPA funded project with UC Davis towards integrating the full encoder/decoder device. Working with UC Davis we have been carrying out the plasma etching of these devices and the results are highlighted below.

The mask layout of the shallowly etched OCDMA (de)coders is shown on the left side of Figure 28. The design has an 8 channels x $200 \mathrm{GHz}$ or a 16 channels x $100 \mathrm{GHz}$ configuration. The central wavelength is $1550 \mathrm{~nm}$ and the free spectral range (FSR) is 16 channels wide for the 8 channel AWG, and 24 channels for the 16 channel AWG. The wide FSR gives rise to high transmission uniformity. The layout also contains sections with straight structures for bends, straight waveguides and phase modulators. The mask has been designed for ridge waveguides that have been etched into the $\mathrm{Q}(1.15)$ core layer partly only, i.e. $0.2 \mu \mathrm{m}$ down from the top of the core layer. The minimum radius of curvature is $500 \mu \mathrm{m}$. Figure 29 shows the output spectrum of the AWG pair (encoder/decoder). 


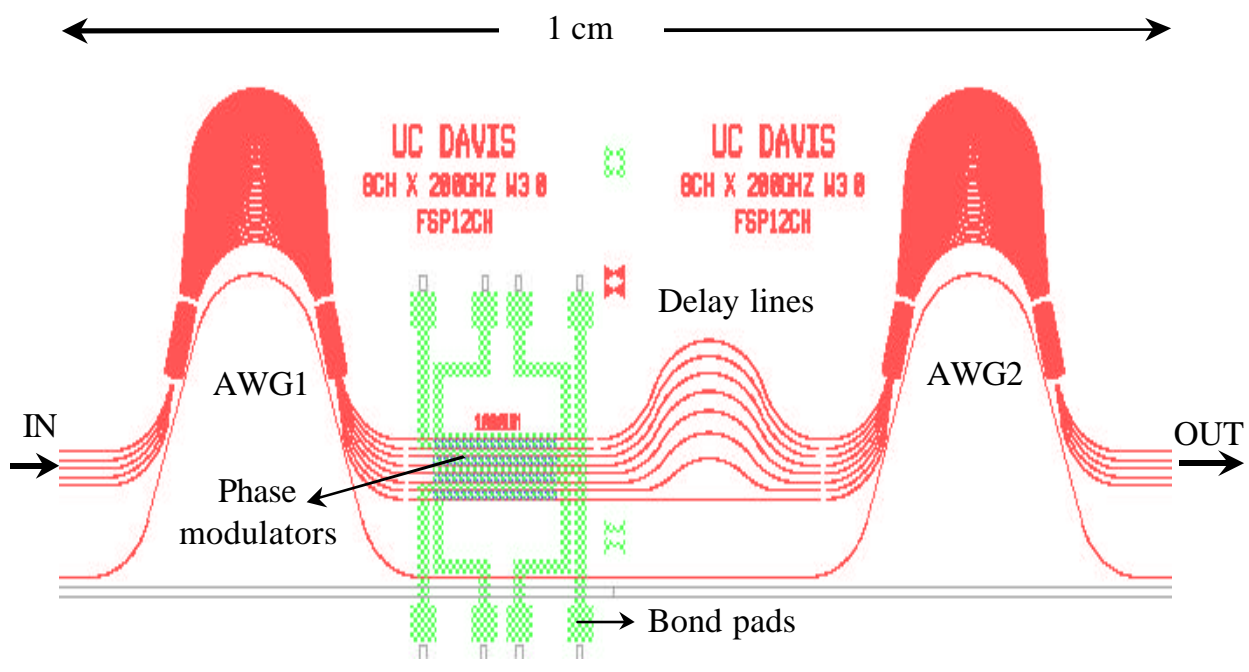

Figure 28: An OCDMA (de)coder chip layout. Phase modulators are 1000um long and delay lines ensure that all eight paths through the modulator have equal optical lengths.

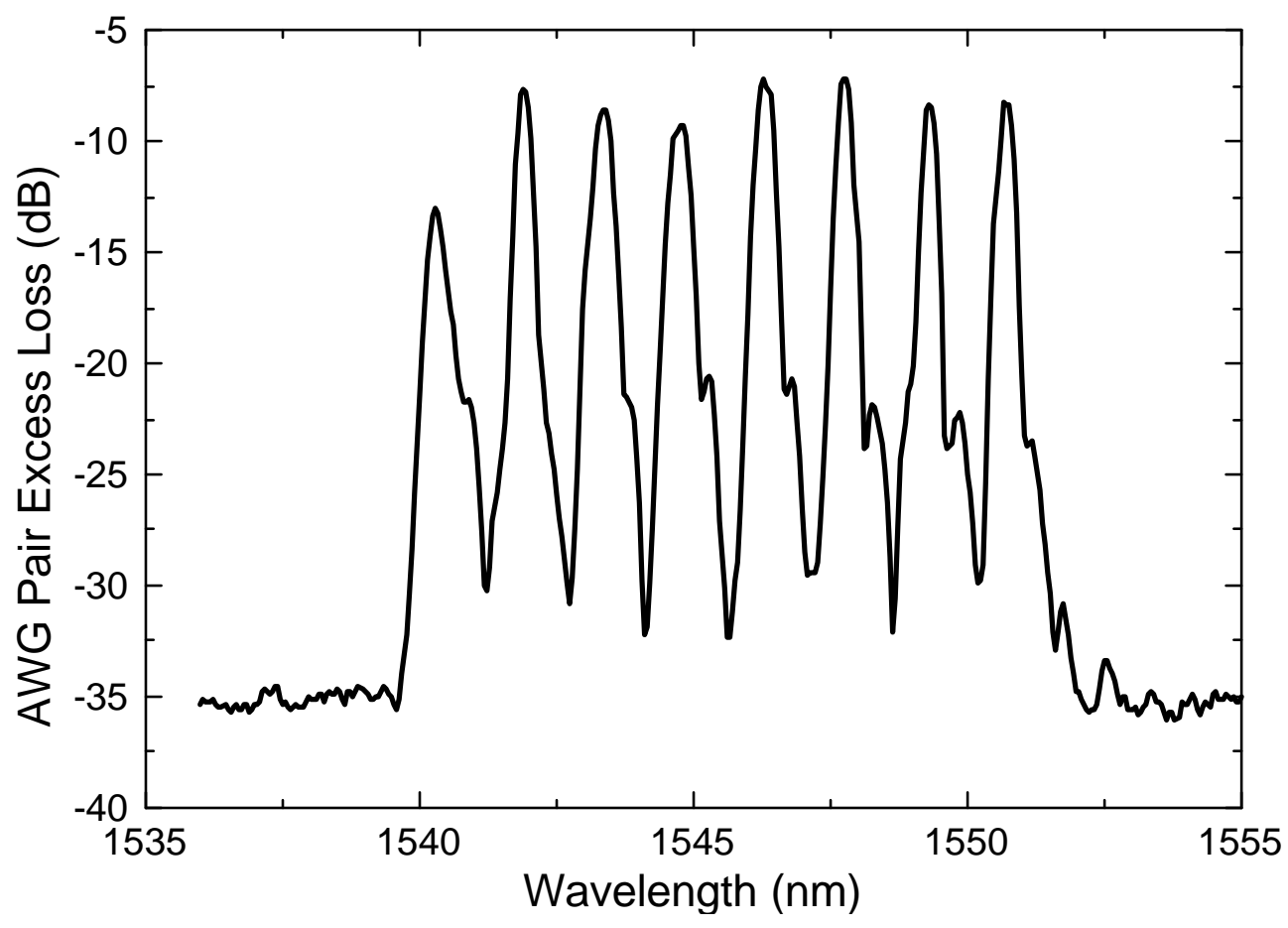

Figure 29: Transmission of pair of AWGs (encoder/decoder). 


\subsection{Exit Plan}

Our exit plan begun in January 2003, with the start of our DARPA sponsored program on OCDMA technologies, of which we are working with University of California at Davis. The DARPA program is aimed at developing a complex semiconductor chip integrating (lasers, phase modulators, waveguides, AWGs and detectors). UCD leads the networks simulation, coding and architecture while LLNL co-leads the InP integrated technology development. Throughout this report we have highlighted the work that is being done in this on-going DARPA project with UC Davis that grew out of our strong collaboration that was started with this LDRD.

\subsection{Summary}

A free space table-top encoder system was designed and evaluated for proof-of-concept for the encoding technique. In parallel our efforts were put towards a substantial investment in setting up the necessary capabilities to fabricate a monolithically integrated encoder device. Our two enabling technologies are semiconductor material growth (metal organic chemical vapor deposition) and plasma etching (electron cyclotron resonance). We developed a novel approach to etching InP based materials using a mixed chlorine and hydrogen plasma. Plasma etching was developed resulting in low loss straight waveguides of $1.2 \mathrm{~dB} / \mathrm{cm}$. We also demonstrated a monolithically integrated arrayed waveguide grating with channel spacing is $3.4 \mathrm{~nm}$ with a signal-to-noise-ration of $20 \mathrm{~dB}$. High density $\mathrm{Cb} / \mathrm{H}_{2}$ based etching, resulting in smooth etched $\mathrm{InP} / \mathrm{InGaAsP}$ surfaces, is a promising technology for the fabrication of photonic integrated circuits which require low loss.

This work has provided the foundation for fabricating a monolithically integrated chip capable of supporting datastreams within a CDMA format within an optical domain. This type of technology maybe required for next generation systems requiring a high level of security, generated by utilizing "locks" and "keys."

[1] J. A. Salehi, A. M. Weiner, and J. P. Heritage, Journal of. Lightwave Technology, vol. 8, p. $547,1990$.

[2] H. P. Sardesai, C.-C. Chang, and A. M. Weiner, Journal of Lightwave Technology, vol. 16, p. 1953, 1998.

[3] A.M. Weiner, J.P. Heritage and E.M. Kirschner, Journal of the Optical Society of America B (Optical Physics), vol.5, no.8, p.1563, Aug. 1988.

[4] C. Drogne, C.A. Edwards, and R.C. Kistler, IEEE Photonics Technology Letters, vol. 3, no. 10 , p. 869, 1991.

[5] H. P. Sardesai, C.-C. Chang, and A. M. Weiner, Journal of Lightwave Technology, vol. 16, p. 1953, 1998.

[6] C. Constantine, C. Barratt, S.J. Pearton, F. Ren, and J.R. Lothian, Applied Physics Letters, vol. 61, no. 24, p. 2899, 1992.

[7] S.J. Choi, K. Djordjev, S.J. Choi, and P.D. Dapkus, Journal of Vacuum Science and Technology B, vol. 20, no. 1, p. 301, 2002.

[8] R. Grover, J.V. Hryniewicz, O.S. King, and V. Van, Journal of Vacuum Science and Technology B, vol. 19, no. 5, p. 1694, 2001.

[9] J.S. Yu, and Y.T. Lee, Journal of the Korean Physical Society, vol. 37, no. 3, p. 241, 2000. 
[10] R.J. Shul and S.J. Pearton, Handbook of Advanced Plasma Processing Techniques, p.478, Springer-Verlag, Berlin, Germany (2000).

[11] R.J. Welty, C.E. Reinhardt, I.Y. Han, Y. Du and S.J. Yoo, International Semiconductor Device Research Symposium, Washington D.C., Dec. 2003.

[12] M. Heinbach, J. Kaindl, and G. Franz, Applied Physics Letters, vol. 67, no. 14, p. 2034, 1995.

[13] L.S. Yu, Q.Z. Liu, S.A. Pappert, P.K.L. Yu, and S.S. Lau, Applied Physics Letters, vol. 64, no. 5, p. 536, 1994. 